\title{
Factors associated with unmet supportive care needs of oncology patients at Dessie Referral Hospital, 2020
}

\author{
Husniya Yasin Amane ${ }^{1}$, Asressie Molla Tessema ${ }^{1}$, Kemal Ahmed seid ${ }^{1}$, Anissa Mohammed Hassen ${ }^{1}$, Hussien Endris Assen ${ }^{2}$, Zinet Abegaz \\ Asfaw $^{1}$, Salih Mohamed endrie ${ }^{3}$ and Foziya Mohammed Hussien ${ }^{1}$
}

${ }^{1}$ Department of Public Health, School of Public Health, College of Medicine and Health Science, Wollo University, Dessie 1145, Ethiopia ${ }^{2}$ Department of Anesthesia and Critical Care, College of Medicine and Health Science, University of Gondar, Gondar 196, Ethiopia

${ }^{3}$ Department of Anesthesia, College of Medicine and Health Science, Wollo University, Dessie 1145, Ethiopia

\section{Abstract}

Background: Assessment of supportive care needs for cancer patients and identifying factors affecting these needs is important for the implementation of supportive care programmes, as the burden of cancer is increasing in Ethiopia.

Objective: To determine the prevalence and associated factors of unmet supportive care needs of cancer patients at Dessie Referral Hospital, Dessie, South Wollo, North East Ethiopia, 2020.

Methods: A cross-sectional study design was implemented among 405 cancer patients from February to 30 July 2020, at Dessie Referral Hospital. The data were collected using a validated supportive care needs survey questionnaire through face to face interview and data extraction tools. Both descriptive and inferential statistics were used and bivariable and multivariable logistic regressions were used to describe the association between dependent and independent variables. Thus, a $p$-value of less than 0.05 was considered statistically significant.

Result: From the total 405 participants, 275 (67.5\%) were females with a mean age of (mean \pm standard deviation) $48.6 \pm 15.4$ years. Unmet supportive care needs were higher among psychological needs (81.0\%, 95\% (confidence interval) $\mathrm{Cl}=77.0-84.9)$ and physical needs $(74.6 \%, 95 \% \mathrm{Cl}=70.1-79.0)$. Old age was associated with unmet physical and psychological needs domain than young age (adjusted odds ratio (AOR) $=1.03 ; 95 \%$ $\mathrm{Cl}: 1.01-1.06),(\mathrm{AOR}=1.06 ; 95 \% \mathrm{Cl}: 1.03-1.09)$, respectively. High household income was significantly associated with health information needs $(\mathrm{AOR}=2.22 ; 95 \% \mathrm{Cl}$ : $1.33-$ 13.93), remission status ( $A O R=0.37 ; 95 \% \mathrm{Cl}: 0.22-0.62$ ) was associated with patient/ supportive care needs, late stage cancer was also significantly associated with physical, psychological and health information needs of patients ( $A O R=2.19 ; 95 \% \mathrm{Cl}: 1.18-4.06$ ), $(\mathrm{AOR}=2.3 ; 95 \% \mathrm{Cl}: 1.18-4.57)$ and $(\mathrm{AOR}=2: 95 \% ; \mathrm{Cl}: 1.03-3.86)$, respectively. Besides, source of information had a statistically significant association with psychological, health information and patient care needs domain (AOR $=2.61 ; 95 \% \mathrm{Cl}: 1.15-5.93)$, (AOR = 3.1; $95 \% \mathrm{Cl}: 1.65-5.82$ ) and ( $\mathrm{AOR}=2.2 ; 95 \% \mathrm{Cl}: 1.25-3.87)$, respectively.
Correspondence to: Anissa Mohammed Hassen Email: anisa.moh26@gmail.com

ecancer 2021, 15:1300

https://doi.org/10.3332/ecancer.2021.1300

Published: 05/10/2021

Received: 22/06/2021

Publication costs for this article were supported by ecancer (UK Charity number 1176307).

Copyright: (C) the authors; licensee ecancermedicalscience. This is an Open Access article distributed under the terms of the Creative Commons Attribution License (http:// creativecommons.org/licenses/by/4.0), which permits unrestricted use, distribution, and reproduction in any medium, provided the original work is properly cited. 
Conclusion and recommendation: This study shows that the prevalence of unmet supportive care needs in cancer patients is high in each domain. Age, income, cancer stage, cancer site, treatment option, time since diagnosis and sources of information were associated across one or more unmet supportive care needs domains. Therefore, the government and health professionals should work together to improve the unmet needs of cancer patients.

Keywords: unmet need, supportive care, oncology, Dessie

\section{Introduction}

In the past few decades, most countries have experienced a health transition that resulted in a dramatic shift in the disease burden from communicable and nutrition-related diseases to non-communicable diseases [1]. Among the global deaths in $2018,63 \%$ were attributed to non-communicable diseases, and the toll is expected to increase further with the ageing of the population, urbanisation and globalisation of risk factors [2].

The global burden of cancer statistics has estimated 7.7 billion new cancer cases and 9.9 billion deaths in 2020 [3]. From the whole cancer data, Europe accounts for $23.4 \%$ of the global cancer cases and $20.3 \%$ of the cancer deaths, but it has only $9.0 \%$ of the worldwide population. On the other hand, the Americas have $13.3 \%$ of the global population and account for $21.0 \%$ of incidence and $14.4 \%$ of mortality worldwide. In contrast to other world regions, the proportions of cancer deaths in Asia and Africa are 57.3\% and 7.3\%, respectively [2]. In Ethiopia, cancer is the second top non-communicable disease next to cardiovascular disorders and its burden is aggravated by lack of early detection and timely treatment $[4,5]$.

The supportive care need is a broad term covering psychological, health system, physical or daily living, patient care or support, and sexuality needs [6]. Due to the disease's progressive nature, supportive care is more important for cancer patients compared with patients with other non-communicable diseases. Various studies have shown that early initiation of supportive care significantly improves the survival and quality of life of cancer patients [7].

'Unmet needs' roughly represents the deficiencies in every area of patients' lives, that arises due to having to deal with a diagnosis of a cancer diagnosis, which lack the level of service or support an individual perceives is necessary to achieve optimal well-being [8]. These needs can develop at any stage in the disease course, from diagnosis to the completion of treatment or death. Assessing the unmet supportive care need of cancer patients has a lot of advantages for both the patient and the government. It helps to prioritise the service to allocate resources depending on the urgency of the need and identify patient subgroups with higher-level needs for prevention or at least reduce problems through appropriate early intervention [9, 10]. According to different research conducted at general cancer population, $27 \%-60.2 \%$ of patients had a low to a high level of unmet supportive care needs [11-13]. Similarly, a study done in the United Kingdom stated that onequarter of the patients reported unmet supportive care needs [14]. According to a survey done in Africa, nearly 46\% of the participants indicated supportive care unmet needs [15].

Unmet needs have been categorised into these major domains: physical, psychological, informational, patient care and sexual needs [16]. Different researchers have found that the largest unmet needs are related to the psychological needs domain [17-19], and in much lesser frequency is found in patient care and sexual domains [20,21]. A systematic review in Asia and Africa showed that psychological, physical and healthcare service/information domains were the three most commonly reported domains of unmet needs for cancer patients [15, 18]. A study conducted at the university of Gondar revealed that the overall mean score level of unmet need was 3.49, with health system and information need being the highest unmet needs [22].

The unmet needs of cancer patients and the level of satisfaction with the overall care were found to influence health related quality of life. Therefore, addressing the unmet needs of cancer patients and ensuring a higher satisfaction rate are recommended to maintain adequate health-related quality of life [23]. Predictors of unmet supportive care needs include younger age, advanced disease, lengthy cancer experience and anticancer treatments. Patients suffer from various problems, such as physical, psychological, emotional and practical issues [24]. 
Currently, the Ethiopian government has made efforts to prevent, control and manage cancer by implementing the Ethiopian National Cancer Control Plan in 2015 for the period of 2016-2020. In addition, the Ministry of Health set up five specialised cancer treatment centres in Gondar, Hawassa, Jimma, Tigray and Southern Nation and Nationality. However, the supportive care of cancer patients has not been considered a health priority and little is known about the supportive care need of oncology patients $[25,26]$. Besides there has been only one study conducted in Ethiopia [22]; therefore, this study aims to determine the prevalence and associated factors of unmet supportive care needs of oncology patients at Dessie referral Hospital (DRH) in the only oncology Centre in North East Ethiopia.

\section{Methods}

\section{Study design and setting}

An institution-based cross-sectional study design was implemented on all cancer patients seen at DRH, Dessie, South Wollo Zone, Ethiopia from February to April 2020. DRH is one of the referral hospitals in the Amhara region with about 9 million catchment populations who came from more than $200 \mathrm{~km}$ away and is used as a teaching hospital for health science students. According to the Centre's cancer registry, the hospital oncology centre started chemotherapy treatment for cancer patients in December 2018 and treated approximately 160 cancer patients per month.

\section{Data collection procedure and participants}

The study populations were all patients diagnosed with cancer on any form of treatment for their disease at Dessie Referral Hospital. Patients who were severely ill and unable to communicate during the data collection period were excluded. Psychological needs, health information needs, physical or daily living, patient care and sexuality needs were the dependent variables. In contrast, socio-demographic characteristics (age, sex, educational status, marital status, employment status, residences, income, health insurances), information related factors (sources of information and informational status) and clinical variables (type of cancer, type of treatment, stage of cancer, time since diagnosis, recurrence history of chronic illness, remission status) were independent variables.

Those cancer patients who came for any type of treatment related to cancer during the data collection period were interviewed using a structured questionnaire adapted from different literatures and supportive care needs survey (SCNS) validated tool (See Appendix 1). The questions include the socio-demographic characteristics, informational status, clinical characteristics and patient's supportive health care needs. Informational status was assessed as a total score composed of 10 different questions, which are basic information received on their diagnosis, prognosis, treatment is taken, medication benefit and side effects, duration of medication, a sequence of treatment, medical and tests a patient undergo the value might range from 0 to 10 maximum response [27, 28]. Data regarding patient's clinical related factors were extracted from a patient card using a chart extraction checklist.

The original SCNS-short form (SF) 34 assesses cancer-specific perceived needs across five domains: Physical and daily living needs, psychological needs, health system information-needs, patient care and support needs and sexuality-needs. The participants were asked to indicate the level of their needs for the last month based on a 5-point Likert scale, The SCNS-SF has been validated at Hawassa referral hospital consisting of 25 items, which had overall Cronbach's alpha of 0.933, ranging from 0.755 to 0.994 for the five domains [29]. For each item, participants could choose either 'not applicable' or 'satisfied' under the heading 'no need', or 'low', 'moderate' or 'high' need under the heading 'some need'.

The data were collected by two BSc nurses working at DRH other than the oncology unit. The quality of data was maintained by giving training to data collectors about the questionnaire's content, collecting data, study design, the significance of the study and the ethics of the research. Continuous monitoring and supervision were conducted by the principal investigator every day for completeness of the data. The questionnaire was translated to Amharic and then translated back to English for consistency. Besides, before the actual data were collected, a pre-test was conducted on $5 \%$ of the sample size for clarity and applicability of the tool, and feedback about the questionnaire. 


\section{Data analysis procedure}

The data were entered and coded into EpiData 4.2 software then exported to SPSS V26 software for analysis. Descriptive statistics and numerical summary measures were presented using frequencies distribution tables and graphs to describe the study population about relevant variables. For continuous variables, we use mean and median if the distribution is normal and skewed, respectively. The outcome variable, supportive health care needs, was recoded into 'no needs' and 'some needs'. If a patient is reported as having at least one low to high need in a domain considered 'unmet needs' in that specific domain, and if a patient reports no need in all items to a single domain, it is considered as 'no needs' [30].

Bivariable logistic regression analysis with the help of odds ratio (OR) along with their $95 \%$ confidence interval (CI) was used to assess the degree of association between dependent and independent variables and variables whose $(p<0.25)$ was a candidate for multivariable logistic regression. Four independent models for each of the SCNS 25 domains were used to determine the association between independent variables and each of the domains (psychological, physical or daily living, health system, supportive needs domain). The statistical significance level was declared at a $p$-value $<0.05$.

\section{Ethical consideration}

This study was approved by the research and ethical committee of Wollo University College of Medicine and Health Science. Informed written consent was also secured from every study participant before the start of the study after telling them about the study's objective. To ensure the confidentiality of the study participant's information, anonymous typing was applied. So, the name of the participant and any identification of participants were not written on the questionnaire. All interviews were taken in a place that keeps privacy, and respondents chose the interview time.

\section{Results}

\section{Socio-demographic characteristics of the participants}

Out of 423 cancer patients seen at DRH during the data collection period, a total of 405 cancer patients aged 18 years or older were enrolled in the study giving a response rate of $95.7 \%$. Of which 275 (67.9\%) were females, and 268 (66.2\%) were married. The mean age was 48.6 years (standard deviation (SD): 15.4) with a minimum of 18 and a maximum of 92 years. Two hundred eleven (52.1\%) were unable to read and write, and 218 (53.8\%) were jobless. More than half (54.1\%) were from an urban area and 233 (57.3\%) had health insurances coverage for their medical expenses.

More than one third of diagnosed cancer cases were breast cancer cases accounting for 138 (34.1\%), followed by cervical cancer cases 77 (19.0\%). Chemotherapy was found to be the leading treatment given for cancer patients accounting for 59.3\%. Three hundred five (75.30\%) were late-stage cancer cases. Eighty-nine (22\%) had a history of remission, and 316 (99\%) had recurrences. Furthermore, eighty-nine (22\%) participants had a history of co-existing disease (Table 1).

Table 1. Socio-demographic and clinical characteristic of oncology patients at Dessie Referral Hospital, Dessie, Ethiopia, 2020.

\begin{tabular}{|l|l|r|}
\hline Variables & Category & \multicolumn{2}{|c|}{ Number of participants $n$ (\%) } \\
\hline Mean age $( \pm$ SD) & \multicolumn{2}{|c|}{$48.6 \pm 15.4$} \\
\hline Sex of the patient & Male & $130(32.1)$ \\
\cline { 2 - 3 } & Female & $275(67.9)$ \\
\hline
\end{tabular}

(Continued) 
Table 1. Socio-demographic and clinical characteristic of oncology patients at Dessie Referral Hospital, Dessie, Ethiopia, 2020. (Continued)

\begin{tabular}{|c|c|c|}
\hline Variables & Category & Number of participants $n(\%)$ \\
\hline \multirow[t]{4}{*}{ Educational status } & Unable to read and write & $211(52.1)$ \\
\hline & Primary education & $97(24.0)$ \\
\hline & Secondary education & $46(11.4)$ \\
\hline & College and above & $51(12.6)$ \\
\hline \multirow[t]{4}{*}{ Occupational status } & Employed & $54(13.3)$ \\
\hline & Merchant & $119(29.4)$ \\
\hline & Jobless/housewife & $218(53.8)$ \\
\hline & Retired & $14(3.5)$ \\
\hline Marital status & $\begin{array}{l}\text { Single } \\
\text { Married } \\
\text { Divorced } \\
\text { Widowed }\end{array}$ & $\begin{array}{c}41(10.1) \\
268(66.2) \\
34(8.4) \\
62(15.3)\end{array}$ \\
\hline Residence & $\begin{array}{l}\text { Rural } \\
\text { Urban }\end{array}$ & $\begin{array}{l}186(45.9) \\
219(54.1)\end{array}$ \\
\hline \multirow[t]{2}{*}{ Health insurance } & Yes & $233(57.5)$ \\
\hline & No & $172(42.5)$ \\
\hline \multirow[t]{4}{*}{ Household income } & $\leq 1,500$ & $155(38.3)$ \\
\hline & $1,501-2,000$ & $91(22.5)$ \\
\hline & $2,001-2,700$ & $60(14.8)$ \\
\hline & $>2,700$ & $99(24.4)$ \\
\hline \multirow[t]{7}{*}{ First cancer site } & Breast & $138(34.1)$ \\
\hline & Colon & $39(9.6)$ \\
\hline & Prostate & $36(8.9)$ \\
\hline & Lung & $37(9.1)$ \\
\hline & Skin & $30(7.4)$ \\
\hline & Cervical & $83(19.0)$ \\
\hline & Lipoma & $42(8.1)$ \\
\hline \multirow[t]{3}{*}{ Treatment options of the patients } & Chemotherapy & $240(59.3)$ \\
\hline & Surgery & $84(20.7)$ \\
\hline & Analgesia & $81(20.0)$ \\
\hline \multirow[t]{4}{*}{ Stage of cancer } & Stage I & $14(3.5)$ \\
\hline & Stage II & $86(21.2)$ \\
\hline & Stage III & $162(40.0)$ \\
\hline & Stage IV & $143(35.3)$ \\
\hline Remission of the disease & Yes & $89(22.0)$ \\
\hline Recurrence of the disease & Yes & $387(99.5)$ \\
\hline Co-existing disease & Yes & $89(22.0)$ \\
\hline Median $\pm I Q R$, time since diagnosis (months) & \multicolumn{2}{|c|}{$4.0 \pm 10$} \\
\hline
\end{tabular}

SD, Standard deviation; IQR, Interquartile range 


\section{Information about diagnosis and related factors}

Of the total patients, 344 (84.9\%) have got information about their diagnosis. Two hundred six (50.9\%) were informed about their current status of a disease. More than half $217(53.6 \%)$ and 247 (61\%) were informed about their possible cause of the disease and the medical test diagnosis of their disease, respectively. Three hundred sixteen (78\%) and 349 (86.2\%) were informed about their medical test and medical result, respectively. Three hundred forty-nine (86.2\%) patients claimed that they were informed about the medical treatment they have taken, and 287 (70.9\%) of patients had information about sequences of treatment they have received. Three hundred four (75.1\%) were informed about the expected benefit, duration and possible side effects of the treatment. More than half 249 (59.5\%) of patients received information from health professionals, and the rest were from health professionals and self-reading. The median and interquartile range (IQR) of patient informational status was $7 \pm 1.6$.

\section{The magnitude of the supportive care needs of participants}

The most frequent need of patients was from the psychological domain accounting for $81 \%$ followed by physical or daily living needs 302 (74.6\%). Finally, health system need was the least unmet supportive care need by patients accounting 87 (21.5\%) (Figure 1).

\section{Factors associated with physical/daily living need domain}

Binary and multivariable logistic regression analysis had been performed to assess the association between the dependent variable (physical needs domain) and independent variables by controlling the confounding variables.

In the binary logistic regression, age, occupational status, marital status, health insurances, household income, cancer site, cancer stage, treatment option, recurrences of diseases and coexisting diseases and informational status were significantly associated with the physical domain; hence further explored in the multivariable logistic analysis. As a result, age, cancer site, treatment option and stage of cancer become important predictors of physical need $(p<0.05)$ (Table 2$)$.

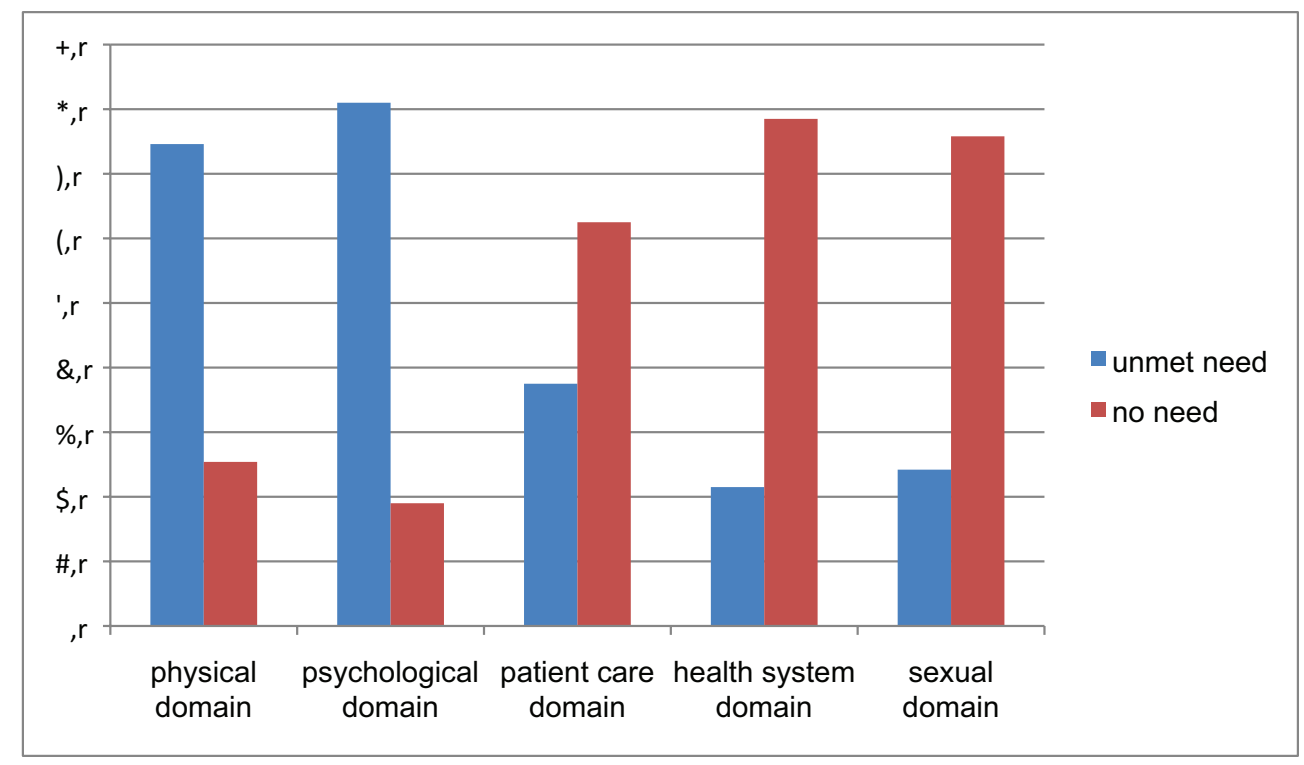

Figure 1. Magnitude of unsupportive care needs of an oncological patient, at Dessie Referral Hospital, Dessie, Ethiopia, 2020. 
Table 2. Binary and multivariable logistic regression of variables associated with physical need domain among oncology patients at DRH, Dessie, Ethiopia, 2020.

\begin{tabular}{|c|c|c|c|c|c|}
\hline \multirow[t]{2}{*}{ Variables } & \multicolumn{5}{|c|}{ Physical need domain } \\
\hline & No need & Some need & $\operatorname{COR}(\mathrm{Cl})$ & AOR (Cl) & $p$ value \\
\hline Age & & & $1.04(1.01-1.05)$ & $1.03(1.01-1.06)$ & $<0.001^{a}$ \\
\hline \multicolumn{6}{|l|}{ Occupational status } \\
\hline Employed & $15(14.6)$ & 39 (12.9) & 1 & 1 & \\
\hline Paid work & $32(31.1)$ & $87(28.8)$ & $1.04(0.51-2.15)$ & $1.33(0.56-3.18)$ & 0.903 \\
\hline Jobless & $55(53.4)$ & $94(54.0)$ & $1.14(0.58-2.22)$ & $1.67(0.66-4.3)$ & 0.701 \\
\hline Retired & $1(1.0)$ & $13(4.3)$ & $5.0(0.6-41.63)$ & $2.62(0.22-30.9)$ & 0.137 \\
\hline \multicolumn{6}{|l|}{ Marital status } \\
\hline Single & $12(11.7)$ & $29(9.6)$ & 1 & 1 & \\
\hline Married & $76(73.8)$ & $192(63.6)$ & $1.04(0.51-15)$ & $0.75(0.30-1.87)$ & 0.904 \\
\hline Divorced & $4(3.9)$ & $30(9.9)$ & $3.1(0.89-10.7)$ & $1.75(0.41-7.51)$ & 0.074 \\
\hline Widowed & $11(10.7)$ & $51(16.9)$ & $1.92(0.75-4.89)$ & $0.92(0.25-3.34)$ & 0.173 \\
\hline \multicolumn{6}{|l|}{ Health insurance } \\
\hline Yes & $69(67.0)$ & $164(54.3)$ & 1 & 1 & \\
\hline No & 34 (33.0) & $138(45.7)$ & $0.58(0.36-0.93)$ & $0.75(0.43-1.34)$ & 0.095 \\
\hline \multicolumn{6}{|c|}{ Monthly household income } \\
\hline $0-1,500$ & $50(48.5)$ & $105(34.8)$ & 1 & 1 & \\
\hline $1,501-2,000$ & $22(21.4)$ & $69(22.8)$ & $1.49(0.83-2.68)$ & $1.82(0.85-3.9)$ & 0.18 \\
\hline $2,001-2,700$ & $9(8.7)$ & $51(16.9)$ & $2.7(1.23-5.91)$ & $2.95(1.19-7.3)$ & $0.013^{\mathrm{a}}$ \\
\hline$>2,700$ & $22(21.4)$ & $77(25.5)$ & $1.66(0.93-2.98)$ & $1.92(0.77-4.8)$ & 0.085 \\
\hline Informational status & \multicolumn{2}{|c|}{-} & $1.18(1.03-1.36)$ & $1.17(0.98-1.38)$ & 0.074 \\
\hline \multicolumn{6}{|l|}{ Cancer site } \\
\hline Breast & $52(50.5)$ & $86(28.5)$ & 1 & 1 & \\
\hline Colon & $10(9.7)$ & $29(9.6)$ & $1.75(0.79-3.89)$ & $1.23(0.49-3.06)$ & 0.167 \\
\hline Prostate & $3(2.9)$ & $33(10.9)$ & $6.6(1.94-22.02)$ & $9.1(2.34-35.74)$ & $0.003^{\mathrm{a}}$ \\
\hline Lung & $2(1.9)$ & $35(11.6)$ & $10.58(2.44-45.8)$ & $8.15(1.76-37.6)$ & $0.002^{\mathrm{a}}$ \\
\hline Skin & $7(6.8)$ & $23(7.6)$ & $1.98(0.79-4.95)$ & $1.67(0.6-4.63)$ & 0.141 \\
\hline Cervical & $18(17.5)$ & $65(21.5)$ & $2.18(1.16-4.08)$ & $2.23(1.06-4.67)$ & $0.014^{\mathrm{a}}$ \\
\hline Lipoma & $11(10.7)$ & $31(10.3)$ & $1.7(0.79-3.67)$ & $1.36(0.54-3.44)$ & 0.174 \\
\hline \multicolumn{6}{|l|}{ Treatment type } \\
\hline Chemotherapy & 75 (72.8) & $165(54.6)$ & 1 & 1 & \\
\hline Surgery & $9(8.7)$ & $75(24.8)$ & $3.7(1.8-7.96)$ & $6.92(2.95-16.2)$ & $<0.001^{\text {a }}$ \\
\hline Analgesia & $19(18.4)$ & $62(20.8)$ & $1.48(0.83-2.65)$ & $1.28(0.64-2.57)$ & 0.184 \\
\hline \multicolumn{6}{|l|}{ Cancer stage } \\
\hline Early stage (I, II) & $38(36.9)$ & $62(20.5)$ & 1 & 1 & \\
\hline Late stage (III, IV) & $65(63.1)$ & $240(79.5)$ & $2.2(1.39-3.69)$ & $2.19(1.18-4.06)$ & $<0.001^{a}$ \\
\hline
\end{tabular}

(Continued) 
Table 2. Binary and multivariable logistic regression of variables associated with physical need domain among oncology patients at DRH, Dessie, Ethiopia, 2020. (Continued)

\begin{tabular}{|c|c|c|c|c|c|}
\hline \multirow[t]{2}{*}{ Variables } & \multicolumn{5}{|c|}{ Physical need domain } \\
\hline & No need & Some need & COR (CI) & $\mathrm{AOR}(\mathrm{Cl})$ & $p$ value \\
\hline \multicolumn{6}{|c|}{ Recurrence } \\
\hline No & $2(1.9)$ & $16(5.3)$ & 1 & 1 & \\
\hline Yes & $101(98.1)$ & $286(94.7)$ & $0.35(0.08-1.56)$ & $0.57(0.11-2.82)$ & 0.171 \\
\hline \multicolumn{6}{|c|}{ Coexisting diseases } \\
\hline No & $88(85.4)$ & $228(75.5)$ & 1 & 1 & \\
\hline Yes & $15(14.6)$ & $74(24.5)$ & $1.9(1.04-3.49)$ & $1.41(0.7-2.86)$ & 0.088 \\
\hline
\end{tabular}

COR, Crude odds ratio; AOR, Adjusted odds ratio; $\mathrm{Cl}$, Confidence interval

a Statistically significant at $p<0.05$

For every 1-year increment in age, the odds of physical unmet needs increases by 3\% (adjusted odds ratio (AOR) = 1.03; $95 \% \mathrm{Cl}: 1.01-1.06)$. The odds of physical needs were nine times more in patients with prostate and eight times more in lung cancer patients than breast cancer patients $(A O R=9.1 ; 95 \% \mathrm{Cl}: 2.34-35.34)$ and (AOR $=8.15 ; 95 \% \mathrm{Cl}: 1.76-37.6)$, respectively. Similarly, the odds of unmet physical needs were six times more in patients undergoing surgery compared with patients taking chemotherapy in the last 1 month (AOR $=6.92$; $95 \% \mathrm{Cl}$ : 2.95-16.2). The odds of unmet physical needs were two times more in patients with end-stage cancer than patients who had early-stage cancer $(\mathrm{AOR}=2.19 ; 95 \% \mathrm{Cl}$ : 1.18-4.06) (Table 2).

\section{Factors associated with psychological need domain}

Binary logistic regression analysis revealed that, age sex, educational level, occupational status, presence of health insurance, household income, sources of information and informational status, cancer site, stage of cancer, treatment option, and time since diagnosis, remission and recurrences of disease were important candidates for the final model. However, only age, cancer site, stage of cancer and source of information become significant predictors in the multivariable logistic regression analysis (Table 3).

Table 3. Binary and multivariable logistic regression of variables associated with psychological need domain among oncology patients at DRH, Dessie, Ethiopia, 2020.

\begin{tabular}{|c|c|c|c|c|c|}
\hline \multirow[t]{2}{*}{ Variables } & \multicolumn{5}{|c|}{ Psychological need } \\
\hline & No need & Some need & $\operatorname{COR}(\mathrm{Cl})$ & AOR (Cl) & $p$ value \\
\hline Age & & & $1.04(1.02-1.06)$ & $1.02(1.01-1.03-1.09)$ & $<0.001$ \\
\hline \multicolumn{6}{|l|}{ Sex } \\
\hline Female & $217(66.2)$ & $58(75.3)$ & 1 & 1 & \\
\hline Male & $111(33.8)$ & $19(24.7)$ & $1.56(0.88-2.75)$ & $0.96(0.46-1.98)$ & 0.123 \\
\hline \multicolumn{6}{|l|}{ Educational status } \\
\hline Unable to read and write & $36(46.8)$ & $175(53.4)$ & 1 & 1 & \\
\hline Primary education & $29(37.7)$ & $68(20.7)$ & $0.48(0.28-0.84)$ & $0.73(0.34-1.56)$ & 0.071 \\
\hline Secondary education & $6(7.8)$ & $40(12.2)$ & $1.37(0.54-3.47)$ & $2.12(0.59-7.53)$ & 0.506 \\
\hline College and above & $6(7.8)$ & 45 (13.7) & $1.54(0.61-3.89)$ & $1.72(0.39-7.51)$ & 0.358 \\
\hline
\end{tabular}

(Continued) 
Table 3. Binary and multivariable logistic regression of variables associated with psychological need domain among oncology patients at DRH, Dessie, Ethiopia, 2020. (Continued)

\begin{tabular}{|c|c|c|c|c|c|}
\hline \multirow[t]{2}{*}{ Variables } & \multicolumn{5}{|c|}{ Psychological need } \\
\hline & No need & Some need & $\operatorname{COR}(\mathrm{Cl})$ & $\operatorname{AOR}(\mathrm{Cl})$ & $p$ value \\
\hline \multicolumn{6}{|c|}{ Household monthly income } \\
\hline $0-1,500$ & $36(46.8)$ & 119 (36.3) & 1 & 1 & \\
\hline $1,501-2000$ & $20(26.0)$ & $71(21.6)$ & $1.07(0.58-1.99)$ & $1.37(0.63-2.97)$ & 0.822 \\
\hline $2,001-2,700$ & $6(7.8)$ & $54(16.5)$ & $2.7(1.08-6.84)$ & $2.23(0.75-6.63)$ & 0.077 \\
\hline$>2,700$ & $15(19.5)$ & $84(25.6)$ & $1.69(0.87-3.29)$ & $1.4(0.49-4.03)$ & 0.120 \\
\hline \multicolumn{6}{|l|}{ Health insurance } \\
\hline Yes & $52(67.5)$ & $181(55.2)$ & 1 & 1 & \\
\hline No & $25(32.5)$ & $147(44.8)$ & $0.59(0.35-1.00)$ & $1.08(0.55-2.09)$ & 0.592 \\
\hline \multicolumn{6}{|l|}{ Source of information } \\
\hline Physician & $50(64.9)$ & $191(58.2)$ & 1 & 1 & \\
\hline Physician and reading & $13(16.9)$ & $85(25.9)$ & $1.71(0.88-3.31)$ & $2.61(1.15-5.93)$ & $0.011^{a}$ \\
\hline \multicolumn{6}{|l|}{ Cancer site } \\
\hline Breast & $39(50.6)$ & $99(30.2)$ & 1 & 1 & \\
\hline Colon & $3(3.9)$ & $36(11.0)$ & $4.7(1.37-16.25)$ & $1.86(0.48-7.23)$ & 0.064 \\
\hline Prostate & $2(2.6)$ & $34(10.4)$ & $6.6(1.53-29.22)$ & $7.17(1.3-39.6)$ & $0.011^{\mathrm{a}}$ \\
\hline Lung & $5(6.5)$ & $32(9.8)$ & $2.56(0.91-6.94)$ & $1.29(0.39-4.22)$ & 0.074 \\
\hline Skin & $4(5.2)$ & $26(7.9)$ & $2.56(0.84-7.81)$ & $1.86(0.52-6.63)$ & 0.099 \\
\hline Cervical & $14(18.2)$ & $69(21.0)$ & $1.94(0.98-3.85)$ & $1.05(0.44-2.5)$ & 0.057 \\
\hline Lipoma & $10(13.0)$ & $32(9.8)$ & $1.26(0.56-2.81)$ & $0.76(0.27-2.13)$ & 0.571 \\
\hline \multicolumn{6}{|l|}{ Treatment option } \\
\hline Chemotherapy & $53(68.8)$ & $187(57.0)$ & 1 & 1 & \\
\hline Surgery & $10(13.0)$ & $74(22.6)$ & $2.09(1.01-4.34)$ & $2.18(0.92-5.21)$ & 0.046 \\
\hline Analgesia & $14(18.2)$ & $67(20.4)$ & $1.35(0.71-2.6)$ & $0.43(0.18-4.57)$ & 0.359 \\
\hline \multicolumn{6}{|l|}{ Cancer stage } \\
\hline Early stage (I, II) & $30(39.0)$ & $70(21.3)$ & 1 & 1 & \\
\hline Late stage (III, IV) & $47(61.0)$ & $258(78.7)$ & $2.35(1.38-3.99)$ & $2.32(1.18-4.57)$ & $0.023^{\mathrm{a}}$ \\
\hline \multicolumn{6}{|l|}{ Recurrence } \\
\hline No & $1(1.3)$ & $17(5.2)$ & 1 & 1 & \\
\hline Yes & $76(98.7)$ & $311(94.8)$ & $0.24(0.03-1.83)$ & $0.3(0.032-2.95)$ & \\
\hline Time since diagnosis & & & $0.98(0.96-1.00)$ & $0.98(0.95-1.01)$ & 0.067 \\
\hline \multicolumn{6}{|l|}{ Remission } \\
\hline No & $10(13.0)$ & $159(48.5)$ & 1 & 1 & \\
\hline Yes & $67(87.0)$ & $169(51.5)$ & $0.16(0.08-0.32)$ & $0.089(0.036-0.22)$ & $<0.001^{a}$ \\
\hline
\end{tabular}

COR, Crude odds ratio; AOR, Adjusted odds ratio; $\mathrm{Cl}$, Confidence interval

aStatistically significant at $p<0.05$ 
For every year increment in age, the odds of unmet psychological needs increased by 6\% (AOR $=1.06$; 95\% Cl: $1.03-1.09$ ). The unmet psychological needs were seven times greater in patients diagnosed with prostate cancer when compared with patients diagnosed with breast cancer (AOR $=7.1 ; 95 \% \mathrm{Cl}: 1.3-39.6)$. Similarly, late-stage cancer patients had 2.3 times unmet psychological needs than early-stage cancer patients ( $A O R=2.32 ; 95 \% \mathrm{Cl}: 1.18-4.57)$. Patients who had sources of information from both health professionals and self-reading had 2.6 times unmet psychological needs than those who had information only from health professionals ( $\mathrm{AOR}=2.61 ; 95 \% \mathrm{Cl}$ : 1.15-5.93). However, the unmet psychological needs were 0.09 times less among patients with a history of remission than no history of remission $(A O R=0.089$; 95\% Cl: 0.036-0.22) (Table 3).

\section{Factors associated with health system information need}

Variables significantly associated in the binary logistic regression were age, occupational status, monthly household income, cancer site, treatment option, stage of cancer, recurrences of diseases and time since diagnosis. However, in the multivariable logistic regression, only treatment option, stage of cancer, higher income and sources of information were significantly associated with health system information needs (Table 4).

The unmet health information needs were $68 \%$ less likely in patients who take analgesia than those taking chemotherapy (AOR $=0.32 ; 95 \%$ $\mathrm{Cl}$ : 0.13-0.82). Similarly, unmet health information needs were two times more in patients who had end-stage cancer than patients who had early-stage cancer ( $\mathrm{AOR}=2$; 95\% Cl: 1.03-3.86). In addition, patients who had household income of $\geq 2,700$ ETB per month had 2.2 times unmet health system need. Moreover, patients who had sources of information from health professionals and self-reading had three times unmet health information needs than those who had information from only health professionals ( $\mathrm{AOR}=3.1 ; 95 \% \mathrm{Cl}: 1.65-5.82)(\mathrm{Table} 4)$.

\section{Factors associated with patient care or supportive need}

First cancer site, remission status, time since diagnosis and sources of information were significant predictors of patient or supportive care needs. The unmet patient care/supportive care needs were $63 \%$ less likely in patients who had a history of remission when compared with patients who had no history of remission ( $A O R=0.37 ; 95 \% \mathrm{Cl}: 0.22-0.62$ ). For every 1 month increase of time since diagnosis, the unmet patient care/supportive needs increased by $2 \%(A O R=1.02 ; 95 \% \mathrm{Cl}$ : 1.00-1.04). Patients who had sources of information from health professionals and reading had more than two times unmet needs than patients who had sources of information only from health professionals $(\mathrm{AOR}=2.2 ; 95 \% \mathrm{Cl}: 1.25-3.87)$. Patients with skin cancer had three times unmet supportive needs than breast cancer patients $(\mathrm{AOR}=2.53$; 95\% Cl: 1.04-6.17) (Table 5).

\section{Discussion}

The study assesses the prevalence and associated factors of unmet supportive care needs of oncology patients at Dessie Referral Hospital. The study revealed that unmet psychological and physical needs were the most unmet supportive care needs domains accounting $81.0 \%$ $(95 \% \mathrm{Cl}=77-84.9)$ and $74.6 \%(95 \% \mathrm{Cl}=70.1-79.0)$, respectively. In addition, old age and household income, cancer site, treatment option, cancer stage, remission, time since diagnosis and sources of information were associated with one or more domains of unmet supportive care need.

The highest unmet supportive care need was recorded under the psychological and physical/daily living domain. Concerns about being able to feel about themselves, anxiety, feelings of sadness and fears about cancer spreading were paramount among unmet needs experienced by patients. The finding is comparable with other studies conducted in Nigeria, United Kingdom, Australia and United Arab Emirates [11, 19, 31, 32]. Emphasising sustained focus on Psycho-oncology treatment and the normal treatment for cancer patients will reduce the unmet needs of cancer patients.

The second top unmet need domain was related to the physical domain, accounting about $74.6 \%$. This is similar to the Iranian study [33]. However, studies conducted in Indonesia and Denmark states as the first top unmet needs accounting (80.4\%) and (40\%), respectively [21, 34]. The explanation could be that cancer treatments, i.e. chemotherapy and surgery have serious side effects like tiredness, vomiting and unable to do normal activities. As a result, this makes them have high physical unmet needs. 
Table 4. Binary and multivariable logistic regression of variables associated with health system information need domain among oncology patients at DRH, Dessie, Ethiopia, 2020.

\begin{tabular}{|c|c|c|c|c|c|}
\hline \multirow[t]{2}{*}{ Variables } & \multicolumn{5}{|c|}{ Health system information need } \\
\hline & No need & Some need & $\operatorname{COR}(\mathrm{Cl})$ & AOR (CI) & $p$ value \\
\hline Age & & & 1.01 (0.99-1.03) & $1.01(0.88-1.04)$ & 0.876 \\
\hline \multicolumn{6}{|l|}{ Occupational status } \\
\hline Employed & $44(13.8)$ & $10(11.5)$ & 1 & 1 & \\
\hline Paid work & $95(30.2)$ & $23(26.4)$ & $1.05(0.46-2.4)$ & $1.32(0.52-3.33)$ & 0.900 \\
\hline Jobless & $169(53.1)$ & $49(56.3)$ & $1.27(0.59-2.72)$ & $1.66(0.58-4.76)$ & 0.528 \\
\hline Retired & $9(2.8)$ & $5(5.7)$ & $2.44(0.67-8.89)$ & $2.62(0.55-12.4)$ & 0.175 \\
\hline \multicolumn{6}{|l|}{ Household income } \\
\hline $0-1,500$ & $112(35.2)$ & $43(49.4)$ & 1 & 1 & \\
\hline $1,501-2,000$ & $78(24.5)$ & $13(14.9)$ & $1.8(1.29-5.86)$ & $0.59(1.27-5.27)$ & 0.002 \\
\hline $2,001-2,700$ & $53(16.7)$ & $7(8)$ & $2.0(1.14-6.81)$ & $1.5(1.15-4.01)$ & 0.003 \\
\hline$>2,700$ & $75(23.6)$ & $24(27.6)$ & $2.56(2.46-9.49)$ & $2.2(1.33-13.93)$ & 0.001 \\
\hline \multicolumn{6}{|l|}{ Source of information } \\
\hline Physician & $207(65.1)$ & 34 (39.1) & 1 & 1 & \\
\hline Physician and reading & $65(20.4)$ & $33(37.9)$ & $3.09(1.78-5.38)$ & $3.06(1.63-5.76)$ & $<0.001^{a}$ \\
\hline \multicolumn{6}{|l|}{ Cancer site } \\
\hline Breast & $106(33.3)$ & $32(36.8)$ & 1 & 1 & \\
\hline Colon & $31(9.7)$ & $8(9.2)$ & $0.85(0.35-2.04)$ & $0.94(0.36-2.41)$ & 0.724 \\
\hline Prostate & 25 (7.9) & $11(12.6)$ & $1.45(0.65-3.28)$ & $1.49(0.57-3.83)$ & 0.363 \\
\hline Lung & $29(9.1)$ & $8(9.2)$ & $0.91(0.38-2.2)$ & $0.9(0.35-2.32)$ & 0.840 \\
\hline Skin & $23(7.2)$ & $7(8.0)$ & $1.00(0.39-2.56)$ & $0.94(0.34-2.6)$ & 0.986 \\
\hline Cervical & $67(21.1)$ & $16(18.4)$ & $0.79(0.4-1.55)$ & $1.26(0.58-2.74)$ & 0.495 \\
\hline Lipoma & $37(11.6)$ & $5(5.7)$ & $0.48(0.16-1.23)$ & $0.46(0.15-1.37)$ & 0.120 \\
\hline \multicolumn{6}{|l|}{ Treatment option } \\
\hline Chemotherapy & $187(58.8)$ & $53(60.9)$ & 1 & 1 & \\
\hline Surgery & $57(17.9)$ & 27 (17.9) & $1.67(0.96-2.89)$ & $1.88(0.98-3.52)$ & 0.067 \\
\hline Analgesia & $74(23.3)$ & $7(8.0)$ & $0.33(0.14-0.77)$ & $0.3(0.12-0.77)$ & $0.01^{a}$ \\
\hline \multicolumn{6}{|l|}{ Cancer stage } \\
\hline Early stage (I, II) & $83(26.1)$ & $17(19.5)$ & 1 & 1 & \\
\hline Late stage (III, IV) & $235(73.9)$ & $70(80.5)$ & $1.45(0.81-2.61)$ & $2(1.03-3.86)$ & $<0.001^{a}$ \\
\hline \multicolumn{6}{|l|}{ Recurrence } \\
\hline No & $10(3.1)$ & $8(9.2)$ & 1 & 1 & \\
\hline Yes & $308(96.9)$ & $79(90.8)$ & $0.32(0.12-0.84)$ & $0.38(0.13-1.12)$ & 0.170 \\
\hline
\end{tabular}

COR, Crude odds ratio; $A O R$, Adjusted odds ratio; $\mathrm{Cl}$, Confidence interval

aStatistically significant at $p<0.05$ 
Table 5. Binary and multivariable logistic regression of variables associated with patient/supportive need domain among oncology patients at DRH, Dessie, Ethiopia, 2020.

\begin{tabular}{|c|c|c|c|c|c|}
\hline \multirow[t]{2}{*}{ Variables } & \multicolumn{5}{|c|}{ Patient care/supportive need } \\
\hline & No need & Some need & COR (CI) & AOR (Cl) & $p$ value \\
\hline \multicolumn{6}{|l|}{ Educational status } \\
\hline Unable to read and write & $135(53.4)$ & $76(50.0)$ & 1 & 1 & \\
\hline Secondary not completed & $61(24.1)$ & $36(23.7)$ & $1.05(0.63-1.72)$ & $1.23(0.67-2.28)$ & 0.853 \\
\hline Secondary education & $29(11.5)$ & $17(11.2)$ & $1.04(0.54-2.01)$ & $0.74(0.31-1.79)$ & 0.905 \\
\hline College and above & $28(11.1)$ & $23(15.1)$ & $1.46(0.78-2.71)$ & $0.87(0.32-2.37)$ & 0.232 \\
\hline \multicolumn{6}{|l|}{ Marital status } \\
\hline Single & $26(10.3)$ & $15(9.9)$ & 1 & 1 & \\
\hline Married & $173(68.4)$ & $95(62.5)$ & $0.95(0.48-1.88)$ & $0.91(0.40-2.08)$ & 0.887 \\
\hline Divorced & $16(6.3)$ & $18(11.8)$ & $0.95(0.77-4.92)$ & $1.5(0.50-4.47)$ & 0.157 \\
\hline Widowed & $38(15.0)$ & $24(15.8)$ & $1.09(0.48-2.47)$ & $1.03(0.35-3.0)$ & 0.828 \\
\hline \multicolumn{6}{|l|}{ Health insurance } \\
\hline Yes & $157(62.1)$ & $76(50.0)$ & 1 & 1 & \\
\hline No & $96(37.9)$ & $76(50.0)$ & $0.61(0.41-0.92)$ & $0.78(0.8-1.25)$ & 0.058 \\
\hline \multicolumn{6}{|l|}{ Household income } \\
\hline $0-1,500$ & $96(37.9)$ & $59(38.8)$ & 1 & 1 & \\
\hline $1,501-2,000$ & $66(26.1)$ & $25(16.4)$ & $0.61(0.35-1.08)$ & $0.65(0.35-1.21)$ & 0.092 \\
\hline $2,001-2,700$ & $36(14.2)$ & $24(15.8)$ & $1.08(0.59-1.99)$ & $1.14(0.56-2.33)$ & 0.794 \\
\hline$>2,700$ & $55(21.7)$ & 44 (28.9) & $1.3(0.78-2.17)$ & $1.31(0.61-2.79)$ & 0.313 \\
\hline \multicolumn{6}{|l|}{ Sources of information } \\
\hline Physician & $159(62.8)$ & $82(53.9)$ & 1 & 1 & \\
\hline Physician and reading & $48(19.0)$ & 50 (32.9) & $2.02(1.05-3.25)$ & $2.2(1.25-3.87)$ & $0.004^{a}$ \\
\hline Mixed & $46(18.2)$ & $20(13.2)$ & $0.84(0.47-1.52)$ & $0.99(0.51-1.91)$ & 0.570 \\
\hline \multicolumn{6}{|l|}{ Cancer site } \\
\hline Breast & $96(37.9)$ & $42(27.6)$ & 1 & 1 & \\
\hline Colon & $25(9.9)$ & $14(9.2)$ & $1.28(0.6-2.7)$ & $1.09(0.48-2.5)$ & 0.518 \\
\hline Prostate & $19(7.5)$ & $17(11.2)$ & $2.04(0.97-4.32$ & $2.07(0.88-4.87)$ & 0.061 \\
\hline Lung & $19(7.5)$ & $17(11.2)$ & $1.74(0.82-3.67$ & $1.72(0.75-3.91)$ & 0.144 \\
\hline Skin & $21(8.3)$ & $16(10.5)$ & $2.0(0.89-4.46)$ & $2.53(1.04-6.17)$ & $0.011^{a}$ \\
\hline Cervical & $50(19.8)$ & $33(21.7)$ & $1.51(0.85-2.67)$ & $1.61(0.82-3.14)$ & 0.157 \\
\hline Lipoma & $26(10.3)$ & $16(10.5)$ & $1.41(0.68-2.89)$ & $1.62(0.71-3.68)$ & 0.353 \\
\hline \multicolumn{6}{|l|}{ Treatment option } \\
\hline Chemotherapy & $154(60.9)$ & $86(56.6)$ & 1 & 1 & \\
\hline Surgery & $45(17.8)$ & $39(25.7)$ & $1.55(0.94-2.57)$ & $1.36(0.76-2.43)$ & 0.087 \\
\hline Analgesia & $54(21.3)$ & $27(17.8)$ & $0.89(0.52-1.52)$ & $0.61(0.32-1.17)$ & 0.684 \\
\hline \multicolumn{6}{|l|}{ Remission status } \\
\hline No & $89(35.2)$ & $80(52.6)$ & 1 & 1 & \\
\hline Yes & $164(64.8)$ & $72(47.4)$ & $0.49(0.32-0.73)$ & $0.37(0.22-0.62)$ & $0.001^{a}$ \\
\hline
\end{tabular}

(Continued) 
Table 5. Binary and multivariable logistic regression of variables associated with patient/supportive need domain among oncology patients at DRH, Dessie, Ethiopia, 2020. (Continued)

\begin{tabular}{|c|c|c|c|c|c|}
\hline \multirow[t]{2}{*}{ Variables } & \multicolumn{5}{|c|}{ Patient care/supportive need } \\
\hline & No need & Some need & $\operatorname{COR}(\mathrm{Cl})$ & AOR (CI) & $p$ value \\
\hline \multicolumn{6}{|l|}{ Recurrence } \\
\hline No & $6(2.4)$ & $12(7.9)$ & 1 & 1 & \\
\hline Yes & $247(97.6)$ & $140(92.1)$ & $0.28(0.1-0.77)$ & $0.34(0.11-1.03)$ & 0.0174 \\
\hline Time since diagnosis & & & 1.01 (0.99-1.03) & 1.02 (1.00-1.04) & $0.042^{\mathrm{a}}$ \\
\hline
\end{tabular}

COR. Crude odds ratio; AOR, Adjusted odds ratio; $\mathrm{Cl}$, Confidence interval

aStatistically significant at $p<0.05$

The least unmet need was related to the sexuality domain. This is in line with studies conducted in Iran [35], Nigeria [32] and Malaysia [36]. However, a study conducted in the United States revealed that the sexuality domain was the most unmet needs of patients [17]. The discrepancy might be due to the cultural, religious and ethical differences of the two countries. Most of the patients in our country are culturally conservative and not ready to disclose information related to sexual behaviour. As a result, further qualitative study is needed to explore the actual sexual needs of the patients. Another reason may be patients may not consider sexual needs as important as other needs like psychological and physical needs.

Our study found that for every increment of age by 1 year, the odds of unmet psychological and physical needs increased by $6 \%$ and $3 \%$, respectively. This is in line with studies conducted in Chicago and England stating older patients had the high unmet needs in physical/daily living and psychological need domain [37, 38]. On the contrary, other researches conducted in the UK and the USA claimed that young age was more prone to unmet supportive health care needs [11,39]. This discrepancy could be explained by old patients are easily fatigued; they cannot resist the side effect of complex and long-term chemotherapy and surgery treatments. As a result, they may develop the unmet physical/psychological needs.

The study also showed high-income patients had high unmet health information needs. Contrary to this, a study done in Athens revealed that high income was less associated with unmet supportive care needs [40]. Economically stable patients may need better treatment options as they can afford the medical fee of private hospitals. However, as the treatment is available at public hospitals, high-income patients may be disappointed by the service delivery compared to private hospitals. Besides, patients who have sources of information from both physician and self-reading have unmet health information need. According to Ethiopia's medical ethical book on Article 27 states, 'On legitimate grounds, left to the discretion of the doctor, information about serious diagnoses and/or prognosis may be withheld unless the patient demands it' [41]. As a result, physicians may hold necessary information related to a disease. So, a patient who had sources of information besides a physician will better understand the prognosis and unfavourable outcome of a disease, and they may develop unmet health system need. Another possibility could be the increasing number of oncology patients leading them to short consultation time with physicians to address all the patients' questions resulting from unmet health information needs.

The current study revealed that prostate cancer patients were more likely to have unmet needs in the physical and psychological domains $(A O R=9.1 ; 95 \% \mathrm{Cl}: 2.34-35.7)$ and $A O R=7.17 ; 95 \% \mathrm{Cl}: 1.3-39.6)$ than breast cancer patients, respectively. Contrarily, a study done in Latin shows that breast cancer survivors reported greater unmet needs compared to both prostate and colorectal cancer survivors (OR 2.33-5.86) [42]. This may be related to most prostate cancer patients having tumour or pain around the gentile area. This makes them psychologically discomfort and unable to move freely to work their normal activities. As a result, patients may need support from another person.

Late-stage cancer patients were two times more likely to have an unmet physical, psychological and health information needs. Similarly in a study done in Malaysia, cancer survivors with an advanced-stage diagnosis had greater physical and psychological needs [43]. This may be related to patients with advanced disease who will have complicated and long-term treatment. The unexpected side effect and struggle for complex and length treatment lead them to have physical and psychological unmet needs. Besides, patients with late-stage cancer will have less survival probability. This finding implies early intervention, and rehabilitation treatment has great clinical importance for cancer patients.

The other variable significantly associated with psychological and patient care/supportive needs is the remission status. Patients with remission were less likely to develop unmet patient care needs $(p<0.001)$. This finding is similar with a Danish population-based study [34]. 
Patients with remission may adapt to the condition of the diseases and hospital environment. Besides, suppose a patient has repeated visits to the hospital. In that case, they may have access to get information and understand their disease condition, making a patient be psychologically ready and decrease their patient care/supportive needs.

Current study states that for every increase of time since diagnosis by 1 month, the odds of the unmet patient care and supportive needs increase by $2 \%$. Similar findings have been reported in an Australian study [44]. The possible reason could be when the time since diagnosis is prolonged, the disease will progress late-stage increasing the severity of the illness. Thus, patients will develop to the verity of the disease increases leading them to develop patient care needs.

Generally, patients treated at DRH had high unmet supportive care needs, especially in psychological, physical and health information needs. Hence, different services and supports that address these needs should be prioritised to fulfil these needs by the patients.

\section{Strengths and limitations}

This is the first study conducted at the only oncology centre of North-East Ethiopia to determine factors associated with unmet supportive care needs of oncology patients. As a result, its representativeness is high in the region. However, due to the study's cross-sectional nature, the study could not show a cause and effect relationship. Besides, excluding patients who are unable to respond due to illness during the data collection may underestimate the specific need of those patients.

\section{Conclusion}

This study revealed that the unmet supportive care needs of a patient within each domain are significantly high. Physical/daily living, psychological and health information/system were the topmost unmet need of cancer patients. In addition, age, income, education status, cancer site, treatment option, stage of cancer, remission and sources of information were associated with increased occurrences of unmet supportive care needs. Thus, the supportive care of cancer patients should be incorporated in the cancer treatment protocol. Furthermore, longitudinal and qualitative researches are better to measure the unmet need of cancer patients at different levels and exploring the unmet need of patients.

\section{Acknowledgments}

The authors' would like to thank Wollo University and the study participants for their willingness to participate in this study.

\section{Consent for publication}

Not applicable.

\section{Availability of data and materials}

All the necessary data are available in the main manuscript document and its supporting information file.

\section{Competing interests}

The authors declare that they have no competing interests. 


\section{Funding}

Wollo University.

\section{Author's contributions}

AM, HY, AM and KA were involved in initiating the idea, write up of the proposal, data collection, data entry, data analysis and final manuscript write up. In contrast, FM, ZA, HE and SE were involved in the final manuscript editing and write up. Finally, all authors were involved in the approval of the final manuscript.

\section{References}

1. United Nation (2015) Transforming Our World: the 2030 Agenda for Sustainable Development (NY: United Nations)

2. Bray F, Ferlay J, and Soerjomataram I, et al (2018) Global cancer statistics 2018: GLOBOCAN estimates of incidence and mortality worldwide for 36 cancers in 185 countries CA Cancer J Clin 68(6) 394-424 https://doi.org/10.3322/caac.21492 PMID: 30207593

3. Sung H, Ferlay J, and Siegel RL, et al (2021) Global cancer statistics 2020: GLOBOCAN estimates of incidence and mortality worldwide for 36 cancers in 185 countries CA Cancer J Clin 71(3) 209-249 https://doi.org/10.3322/caac.21660 PMID: 33538338

4. Pisani $P$ (2011) The cancer burden and cancer control in developing countries Environ Health 10(1) S2 https://doi.org/10.1186/1476069X-10-S1-S2 PMID: 21489212 PMCID: 3073194

5. Misganaw A, Mariam DH, and Ali A, et al (2014) Epidemiology of major non-communicable diseases in Ethiopia: a systematic review J Health Popul Nutr 32 1-13 PMID: 24847587 PMCID: 4089066

6. McElduff P, Boyes A, and Zucca A, et al (2004) Supportive Care Needs Survey: a Guide to Administration, Scoring and Analysis (Newcastle: Centre for Health Research \& Psycho-Oncology)

7. NICE (2004) Improving Supportive and Palliative Care for Adults with Cancer: Executive Summary (UK: National Institute for Clinical Excellence)

8. Fitch M (200) Supportive care for cancer patients Hosp Q 3(4) 39-46 PMID: 11482268

9. Bonevski B, Sanson-Fisher R, and Girgis A, et al (2000) Evaluation of an instrument to assess the needs of patients with cancer Cancer 88(1) 217-225 https://doi.org/10.1002/(SICI)1097-0142(20000101)88:1\&It;217::AID-CNCR29\&gt;3.0.CO;2-Y PMID: 10618626

10. Alananzeh IM, Levesque JV, and Kwok C, et al (2019) The unmet supportive care needs of Arab Australian and Arab Jordanian cancer survivors: an international comparative survey Cancer Nurs 42(3) E51-E60 https://doi.org/10.1097/NCC.0000000000000609

11. Molassiotis A, Brunton L, and Hodgetts J, et al (2014) Prevalence and correlates of unmet supportive care needs in patients with resected invasive cutaneous melanoma Ann Oncol 25(10) 2052-2058 https://doi.org/10.1093/annonc/mdu366 PMID: 25081900

12. Wang S, Li Y, and Li C, et al (2018) Distribution and determinants of unmet need for supportive care among women with breast cancer in China Med Sci Monit 24 1680-1687 https://doi.org/10.12659/MSM.905282 PMID: 29561832 PMCID: 5877206

13. Boyes AW, Girgis A, and D'Este C, et al (2012) Prevalence and correlates of cancer survivors' supportive care needs 6 months after diagnosis: a population-based cross-sectional study BMC Cancer 12(1) 150 https://doi.org/10.1186/1471-2407-12-150

14. McGarry S, Ward C, and Garrod R, et al (2013) An exploratory study into the unmet supportive needs of breast cancer patients Eur J Cancer Care 22(5) 673-683 https://doi.org/10.1111/ecc.12076 
15. Fatiregun O, Sowunmi AC, and Habeebu M, et al (2019) Prevalence and correlates of unmet supportive needs of Nigerian patients with cancer J Glob Oncol 5 1-9

16. Kerr LM, Harrison MB, and Medves J, et al (2004) Supportive care needs of parents of children with cancer: transition from diagnosis to treatment Toronto: ontario cancer treatment and research foundation Oncol Nurs Forum 13 116-126 https://doi.org/10.1188/04.ONF. E116-E126

17. Gonzalez V, Velez M, and Pedro E, et al (2009) Identification of supportive care needs in a sample of Puerto Rican cancer patients with the Supportive Care Needs Survey-34 (SCNS-34) J Clin Oncol 27(15_suppl) e20697 https://doi.org/10.1200/jco.2009.27.15_suppl. e20697

18. Wang T, Molassiotis A, and Chung BPM, et al (2018) Unmet care needs of advanced cancer patients and their informal caregivers: a systematic review BMC Palliat Care 17(1) 96 https://doi.org/10.1186/s12904-018-0346-9 PMID: 30037346 PMCID: 6057056

19. Minstrell M, Winzenberg T, and Rankin N, et al (2008) Supportive care of rural women with breast cancer in Tasmania, Australia: changing needs over time Psychooncology 17(1) 58-65 https://doi.org/10.1002/pon.1174

20. Pérez-Fortis A, Fleer J, and Sánchez-Sosa JJ, et al (2017) Prevalence and factors associated with supportive care needs among newly diagnosed Mexican breast cancer patients Support Care Cancer 25(10) 3273-3280 https://doi.org/10.1007/s00520-017-3741-5 PMID: 28516220 PMCID: 5577048

21. Afiyanti Y, Milanti NA, and Putri RH (2018) Supportive care needs in predicting the quality of life among gynecological cancer patients Can Oncol Nurs 28(1) 22 https://doi.org/10.5737/236880762812229

22. Gebresillassie BM, Ayele AA, and Abegaz TM (2020) Unmet supportive care needs and determinants among cancer patients treated at University of Gondar Specialized Hospital, Northwest Ethiopia: a prospective cross-sectional study J Oncol Pharm Pract https://doi. org/10.1177/1078155220971035 PMID: 33148130

23. Abegaz TM, Ayele AA, and Gebresillassie BM (2018) Health related quality of life of cancer patients in Ethiopia J Oncol $2018 \mathrm{https}: / /$ doi. org/10.1155/2018/1467595 PMID: 29849628 PMCID: 5925207

24. Okediji P, Salako O, and Fatiregun $\mathrm{OO}$ (2018) Pattern and predictors of unmet supportive care needs in cancer patients Ame Soc Clin Oncol

25. Ferlay J, Soerjomataram I, and Ervik $\mathrm{M}$ et al (2015) Cancer incidence and mortality worldwide: sources, methods and major patterns in GLOBOCAN 2012 Int J Cancer 136(5) E356-86

26. WHO (2019) United Nations high-level meeting on noncommunicable disease prevention (Geneva: WHO)

27. Ardestani SMS, Faridhosseini F, and Shirkhani F, et al (2015) Do cancer patients prefer to know the diagnosis? A descriptive study among Iranian patients Iran J Psychiatry Behav Sci 9(4) e1792

28. Laxmi S, and Khan JA (2013) Does the cancer patient want to know? Results from a study in an Indian tertiary cancer center South Asian J Cancer 2(2) 57-61 https://doi.org/10.4103/2278-330X.110487

29. Afework T, Wondimagegnehu A, and Alemayehu N, et al (2021) Validity and reliability of the Amharic version of supportive care needs survey-short form 34 among cancer patients in Ethiopia BMC Health Serv Res 21(1) 1-10 https://doi.org/10.1186/s12913-021-06512-2

30. McElduff P, Boyes A, and Zucca A, et al (2004) Supportive care needs survey: a guide to administration, scoring and analysis

31. Nair SC, Jaafar H, and Jaloudi M, et al (2018) Supportive care needs of multicultural patients with cancer in the United Arab Emirates Ecancermedicalscience 12838 PMID: 29910835 PMCID: 5985753

32. Fatiregun O, Okediji P, and Awofeso O, et al (2018) The unmet supportive care needs of Nigerian patients with cancer Am Soc Cli Oncol 
33. Faghani S, Mohammadian R, and Rahmani A, et al (2015) Supportive care needs of Iranian cancer survivors and relationships with social support Asian Pac J Cancer Prev 16(15) 6339-6345 https://doi.org/10.7314/APJCP.2015.16.15.6339 PMID: 26434840

34. Hansen DG, Larsen PV, and Holm LV, et al (2013) Association between unmet needs and quality of life of cancer patients: a populationbased study Acta Oncol 52(2) 391-399 https://doi.org/10.3109/0284186X.2012.742204

35. Fong EJ, and Cheah WL (2016) Unmet supportive care needs among breast cancer survivors of community-based support group in Kuching, Sarawak Int J Breast Cancer 2016 https://doi.org/10.1155/2016/7297813 PMID: 27239346 PMCID: 4863116

36. Edib Z, Kumarasamy V, and binti Abdullah N, et al (2016) Most prevalent unmet supportive care needs and quality of life of breast cancer patients in a tertiary hospital in Malaysia Health Qual Life Outcomes 14(1) 1-10 https://doi.org/10.1186/s12955-016-0428-4

37. Boberg EW, Gustafson DH, and Hawkins RP, et al (2003) Assessing the unmet information, support and care delivery needs of men with prostate cancer Patient Educ Couns 49(3) 233-242 https://doi.org/10.1016/S0738-3991(02)00183-0 PMID: 12642195

38. Ream E, Quennell A, and Fincham L, et al (2008) Supportive care needs of men living with prostate cancer in England: a survey Brit J Cancer 98(12) 1903-1909 https://doi.org/10.1038/sj.bjc.6604406 PMID: 18506142 PMCID: 2441950

39. Bonevski B, Sanson-Fisher R, and Hersey P, et al (2000) Assessing the perceived needs of patients attending an outpatient melanoma clinic J Psychosoc Oncol 17(3-4) 101-118 https://doi.org/10.1300/J077v17n03_06

40. Psychogyiou A, Katsaragakis S, and Lemonidou C, et al (2018). CN32 Most prevalent unmet supportive care needs in Greek ambulatory advanced breast cancer female patients receiving chemotherapy Ann Oncol 29(suppl_8) mdy341. 031 https://doi.org/10.1093/annonc/ mdy341.031

41. Ethiopian Medical Association (2016) Medical ethics for doctors in Ethiopia (Ethiopia: Ethiopian Medical Association (EMA))

42. Moreno PI, Ramirez AG, and San Miguel-Majors SL, et al (2019) Unmet supportive care needs in Hispanic/Latino cancer survivors: prevalence and associations with patient-provider communication, satisfaction with cancer care, and symptom burden Support Care Cancer 27(4) 1383-1394 https://doi.org/10.1007/s00520-018-4426-4 PMCID: 6386634

43. Edib Z, Kumarasamy V, and Binti Abdullah N, et al (2016) Most prevalent unmet supportive care needs and quality of life of breast cancer patients in a tertiary hospital in Malaysia Health Qual Life Outcomes 14(1) 26 https://doi.org/10.1186/s12955-016-0428-4 PMID: 26898558 PMCID: $\underline{4762172}$

44. Sutherland G, Hill D, and Morand M, et al (2009) Assessing the unmet supportive care needs of newly diagnosed patients with cancer Eur J Cancer Care 18(6) 577-584 https://doi.org/10.1111/j.1365-2354.2008.00932.x 


\section{Appendix 1}

\section{Information sheet}

Name of the investigator: Husniya Yasin

Name of the organization: Wollo University

\section{Introduction:}

This information sheet is prepared by the investigator from wollo university, department of public health whose main aim is to assess the prevalence of unmet needs of oncologic patients and factors associated with the unmet health care needs of oncology patients.

\section{Purpose of the research}

the purpose of this research is to determine factors associated with unmet health care need of oncology patient.

\section{Voluntary participation}

Your participation in this research is entirely voluntary. It is your choice whether to participate or not. Whether you choose to participate or not, all the services you receive as any member of this community will continue and nothing will change. If you choose not to participate in this research, you will be offered all the services that are routinely offered. You may change your mind later and stop participating even if you agreed earlier.

\section{Confidentiality}

The information collected for this research will be kept confidential. Information about you that is collected during the research will be put away and no one but the researcher will be able to see it. Any information about you will have a number on it instead of your name. Only the researchers will know what your number is and Keep that information very secret that no one else can access, see or know it. It will not be shared with anyone.

\section{Benefits}

this research may benefit you directly as an individual but it may have a benefit for health facilities for intervention.

\section{Risks and side effects}

There are no side effects and known risks related to this of research so far.

\section{Who to contact}

This research will be reviewed and approved by the ethical review committee of Wollo University. If you wish to find about more or if you wish to ask questions now or later you can use the contact addresses below

Husniya Yasin 0913196525 


\section{Consent Form}

\section{Greeting:}

My name is

I am here to collect information from you to know the health care need. Your participation in this research is entirely voluntary. It is your choice whether to participate or not. Whether you choose to participate or not, all the services you receive as any member of this community will continue and nothing will change. Information about you that is collected during the research will be put away and no one but the researcher will be able to see it. Your participation in this research may not directly provide you a certain benefit as an individual. It may benefit all mothers and children. There are no side effects and Known risks related to this kind of research so far and it takes only 10 minutes of participation. Up to now, you have been given all information that I feel you should know regarding the research project that you are being asked to participate in. I think you have understood the issues in detail. As I told you the survey has no risk, confidential, and takes only 10 minutes of interview.

Thank you for your cooperation and listening!!!

Are you willing to participate?

Yes No (stop the interview)

Name of data collector signature

Name of Supervisor signature

\section{Questionnaire}

Code number

Date

\section{Part I Socio-demographic characteristics}

\begin{tabular}{|c|c|c|}
\hline Q.NO & QUESTIONNARIES & RESPONSE \\
\hline 01 & Age & -------------- \\
\hline 02 & Sex & $\begin{array}{l}\text { 1. male } \\
\text { 2. Femalle }\end{array}$ \\
\hline 03 & Marital status & $\begin{array}{l}\text { 1. single } \\
\text { 2. married } \\
\text { 3. Divorced/separated } \\
\text { 4. Widowed } \\
\text { 5. Never married/single }\end{array}$ \\
\hline 04 & Educational Level & $\begin{array}{l}\text { 1. anable to write and read } \\
\text { 2. Secondary not completed } \\
\text { 3. Secondary completed } \\
\text { 4. Trade/certificate/diploma } \\
\text { 5. Tertiary completed }\end{array}$ \\
\hline
\end{tabular}




\begin{tabular}{|c|l|l|}
\hline Q.NO & QUESTIONNARIES & RESPONSE \\
\hline 05 & occupation & $\begin{array}{l}\text { 1. employment } \\
\text { 2. Paid work } \\
\text { 3. Not working } \\
\text { 4. Retired }\end{array}$ \\
\hline 06 & Income & ------------- \\
\hline 07 & Resident & $\begin{array}{l}\text { 1. Urban } \\
\text { 2. Rural }\end{array}$ \\
\hline
\end{tabular}

\section{Part II Clinical Characteristics}

\begin{tabular}{|c|c|c|}
\hline 07 & Primary cancer site & $\begin{array}{l}\text { 1. Breast } \\
\text { 2. Colon and rectum } \\
\text { 3. Prostate } \\
\text { 4. Lung } \\
\text { 5. Skin/melanoma } \\
\text { 6. Don't know } \\
\text { 7. Other }\end{array}$ \\
\hline 08 & Treatment received in last month & $\begin{array}{l}\text { 1. Chemotherapy } \\
\text { 2. Radiotherapy } \\
\text { 3. Surgical removal of cancer } \\
\text { 4. Immunotherapy } \\
\text { 5. Hormone treatment } \\
\text { 6. Bone marrow treatment } \\
\text { 7. Othe }\end{array}$ \\
\hline 09 & Time since diagnosis & - \\
\hline 010 & Remission & $\begin{array}{l}\text { 1. yes } \\
\text { 2. no }\end{array}$ \\
\hline 011 & Another illness other than cancer & $\begin{array}{l}\text { 1. yes } \\
\text { 2. no }\end{array}$ \\
\hline 012 & Type of diseases & \\
\hline 012 & Stage of cancer at time of diagnosis & $\begin{array}{l}\text { 1. I } \\
\text { 2. II } \\
\text { 3. III } \\
\text { 4. IV } \\
\text { 5. Unknown }\end{array}$ \\
\hline 013 & Fear of recurrences & $\begin{array}{l}\text { 1. yes } \\
\text { 2. no }\end{array}$ \\
\hline 014 & Treatment side effect & $\begin{array}{l}\text { 1. yes } \\
\text { 2. no }\end{array}$ \\
\hline
\end{tabular}




\section{Part III Information status about patient diagnosis}

\begin{tabular}{|c|c|c|c|}
\hline No & Questions & Coding categories & Skip \\
\hline \multicolumn{4}{|c|}{ During your current disease or treatment, have you received information on: } \\
\hline 01 & The diagnosis of your disease? & $\begin{array}{l}\text { 1. Yes. } \\
\text { 2. No }\end{array}$ & \\
\hline 02 & The extent (spread) of your disease? & $\begin{array}{l}\text { 1. Yes. } \\
\text { 2. No }\end{array}$ & \\
\hline 03 & The possible causes of your disease? & $\begin{array}{l}\text { 1. Yes. } \\
\text { 2. No }\end{array}$ & \\
\hline 04 & The purpose of any medical tests you have had or may undergo & $\begin{array}{l}\text { 1. Yes. } \\
\text { 2. No }\end{array}$ & \\
\hline 05 & The procedures of the medical tests? & $\begin{array}{l}\text { 1. Yes. } \\
\text { 2. No }\end{array}$ & \\
\hline 06 & $\begin{array}{l}\text { The medical treatment (chemotherapy, radiotherapy, surgery or other } \\
\text { treatment modality)? }\end{array}$ & $\begin{array}{l}\text { 1. Yes. } \\
\text { 2. No }\end{array}$ & \\
\hline 07 & The sequence of the medical treatments? & $\begin{array}{l}\text { 1. Yes. } \\
\text { 2. No }\end{array}$ & \\
\hline 08 & The expected benefit of the treatment? & $\begin{array}{l}\text { 1. Yes. } \\
\text { 2. No }\end{array}$ & \\
\hline 09 & The possible side-effects of your treatment? & $\begin{array}{l}\text { 1. Yes. } \\
\text { 2. No }\end{array}$ & \\
\hline 010 & Where did you inform about the duration of your treatments? & $\begin{array}{l}\text { 1. Yes. } \\
\text { 2. No }\end{array}$ & \\
\hline 011 & $\begin{array}{l}\text { From whom did you hear about your diagnosis? } \\
\text { Record all mentioned }\end{array}$ & $\begin{array}{l}\text { 1. health professional } \\
\text { 2. from a family member } \\
\text { 3. other patients } \\
\text { 4. other }\end{array}$ & \\
\hline
\end{tabular}

\section{Part IV supportive care need tool}

Instructions: This section of the questioner will assess supportive care needs, please circle the numbers or record on the space provided under the coding categories

\begin{tabular}{|c|c|c|c|c|c|c|}
\hline \multirow{2}{*}{\multicolumn{2}{|c|}{ Physical need domain ( 5 items) }} & \multicolumn{5}{|c|}{ Coding category } \\
\hline & & \multicolumn{2}{|c|}{ No need } & \multicolumn{3}{|c|}{ Unmet need } \\
\hline No & & Not applicable & satisfied & Low need & Moderat need & High need \\
\hline 1 & Pain & 1 & 2 & 3 & 4 & 5 \\
\hline 2 & Lack of energy/tiredness & 1 & 2 & 3 & 4 & 5 \\
\hline 3 & Feeling unwell most of the time & 1 & 2 & 3 & 4 & 5 \\
\hline 4 & Work around the home & 1 & 2 & 3 & 4 & 5 \\
\hline 5 & Not being able to do the thing you used to do & 1 & 2 & 3 & 4 & 5 \\
\hline
\end{tabular}




\begin{tabular}{|c|c|c|c|c|c|c|}
\hline \multirow{3}{*}{\multicolumn{2}{|c|}{ Psychological domain }} & \multicolumn{5}{|c|}{ Coding category } \\
\hline & & \multicolumn{2}{|c|}{ No need } & \multicolumn{3}{|c|}{ Unmet need } \\
\hline & & Not applicable & satisfied & Low need & Moderat need & High need \\
\hline 1 & Anxiety & 1 & 2 & 3 & 4 & 5 \\
\hline 2 & Feeling down or depression & 1 & 2 & 3 & 4 & 5 \\
\hline 3 & Feeling of sadness & 1 & 2 & 3 & 4 & 5 \\
\hline 4 & Fear about the cancer spreading & 1 & 2 & 3 & 4 & 5 \\
\hline 5 & $\begin{array}{l}\text { Worry that the results of treatment are beyond your } \\
\text { control }\end{array}$ & 1 & 2 & 3 & 4 & 5 \\
\hline 6 & Uncertainty about the future & 1 & 2 & 3 & 4 & 5 \\
\hline 7 & Learning to feel in control of your situation & 1 & 2 & 3 & 4 & 5 \\
\hline 8 & Keeping a positive outlook & 1 & 2 & 3 & 4 & 5 \\
\hline 9 & Feeling about death and dying & 1 & 2 & & 4 & 5 \\
\hline 10 & Concern about the worries of those to you & 1 & 2 & 3 & 4 & 5 \\
\hline \multirow{2}{*}{\multicolumn{2}{|c|}{ Patient care/supportive need ( 3 items) }} & \multicolumn{2}{|c|}{ No need } & \multicolumn{3}{|c|}{ Some need } \\
\hline & & Not applicable & satisfied & Low need & $\begin{array}{c}\text { Moderate } \\
\text { need }\end{array}$ & High need \\
\hline 1 & Reassurance by medical staff the way you feel is normal & 1 & 2 & 3 & 4 & 5 \\
\hline 2 & Hospital staff attending promptly to your physical need & 1 & 2 & 3 & 4 & 5 \\
\hline 3 & $\begin{array}{l}\text { Hospital staff acknowledging and showing sensitivity to } \\
\text { your feeling and emotional needs }\end{array}$ & 1 & 2 & 3 & 4 & 5 \\
\hline \multirow{2}{*}{\multicolumn{2}{|c|}{ Health system/information (4 items) }} & \multicolumn{2}{|c|}{ No need } & \multicolumn{3}{|c|}{ Some need } \\
\hline & & Not applicable & satisfied & Low need & Moderat need & High need \\
\hline 1 & $\begin{array}{l}\text { To be given explanations of those } \\
\text { tests for which you would like } \\
\text { explanations }\end{array}$ & 1 & 2 & 3 & 4 & 5 \\
\hline 2 & $\begin{array}{l}\text { To be adequately informed about } \\
\text { the benefits and side effects of } \\
\text { treatments before you choose to } \\
\text { have them }\end{array}$ & 1 & 2 & 3 & 4 & 5 \\
\hline 3 & $\begin{array}{l}\text { To be informed about your test } \\
\text { results as soon as possible }\end{array}$ & 1 & 2 & 3 & 4 & 5 \\
\hline 4 & $\begin{array}{l}\text { To be informed about cancer that is } \\
\text { under control or diminishing (that } \\
\text { is, remission) }\end{array}$ & 1 & 2 & 3 & 4 & 5 \\
\hline \multirow{3}{*}{\multicolumn{2}{|c|}{ Sexual domain (3 item) }} & \multicolumn{5}{|c|}{ Coding category } \\
\hline & & \multicolumn{2}{|c|}{ No need } & \multicolumn{3}{|c|}{ Some need } \\
\hline & & Not applicable & Satisfied & Low need & Moderat need & High need \\
\hline 1 & Change in sexual relationships & & & & & \\
\hline 2 & Change in sexual feeling & & & & & \\
\hline 3 & $\begin{array}{l}\text { To be given information about } \\
\text { Sexual relationships }\end{array}$ & 1 & 2 & 3 & 4 & 5 \\
\hline
\end{tabular}




\section{Amharic Version Questionnaire}

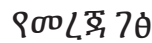

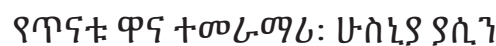

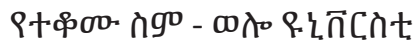

00\%ก.9

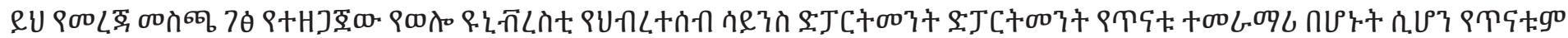

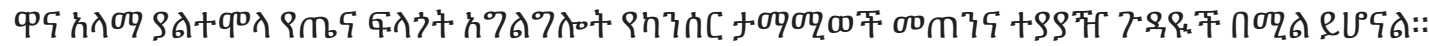

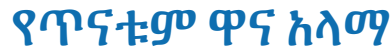

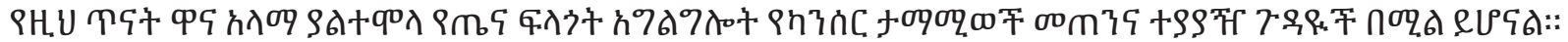

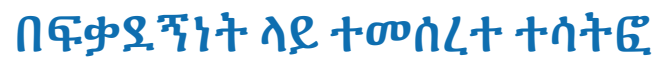

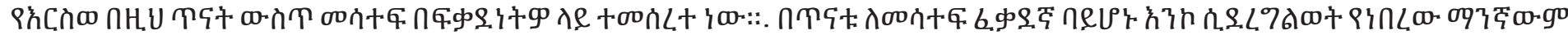

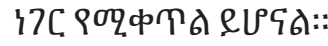

$00+0900$ F

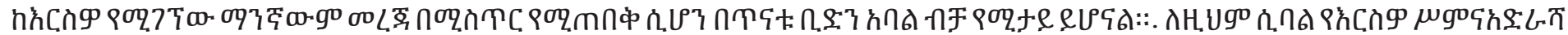

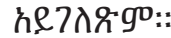

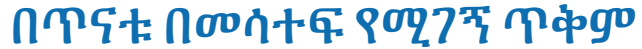

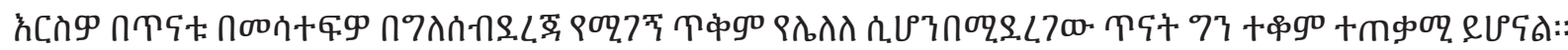

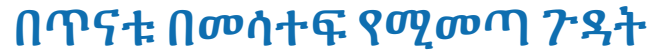

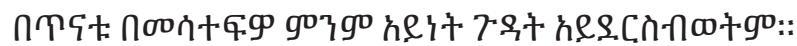

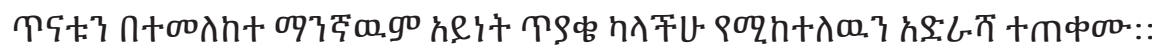

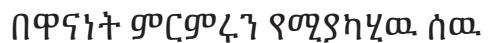

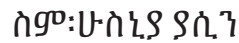


9กล่ 中ก 0913196525

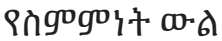

ก่ $\lambda 9^{\circ}+$

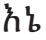

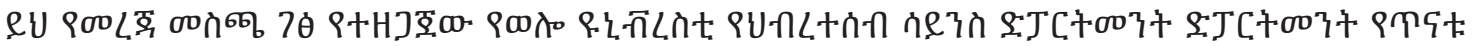

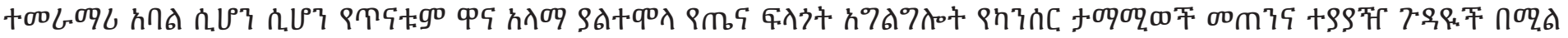

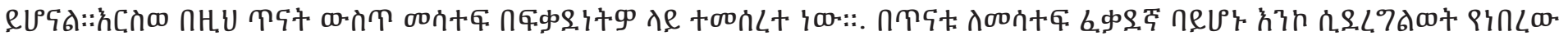

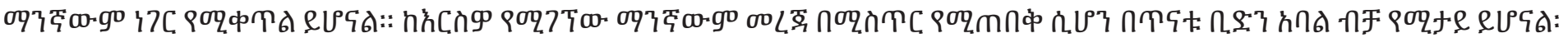

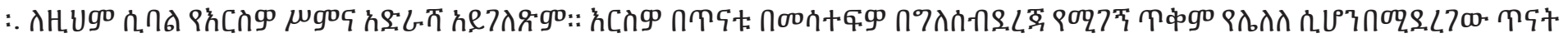

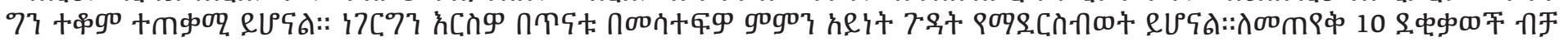

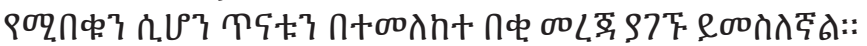

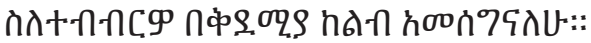

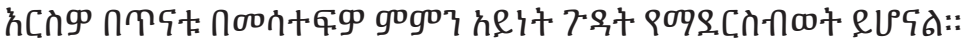

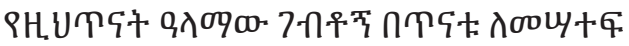

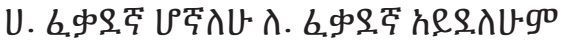

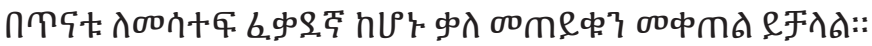

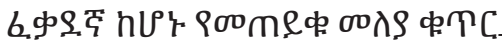
ovmed $9+h 4 \Omega \cap+\phi ?$

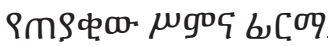

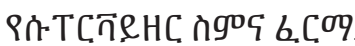

\section{ovm}

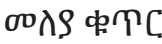

\$?

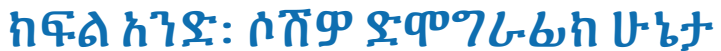

\begin{tabular}{|c|c|c|}
\hline †.q & Ћ९фळ军 & סָ חָ \\
\hline 01 & & \\
\hline 02 & 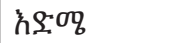 & _-_ \\
\hline 03 & $9+$ & $\begin{array}{l}1.039 \\
2.07\end{array}$ \\
\hline 04 & ९コクモひな & 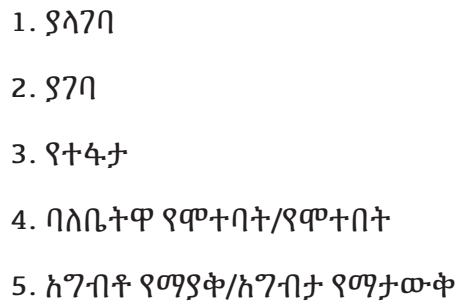 \\
\hline
\end{tabular}




\begin{tabular}{|c|c|c|}
\hline +. $\$$ & ๆ९ф্口市 & סטגה \\
\hline 05 & $9+90 /+9 L S$ & 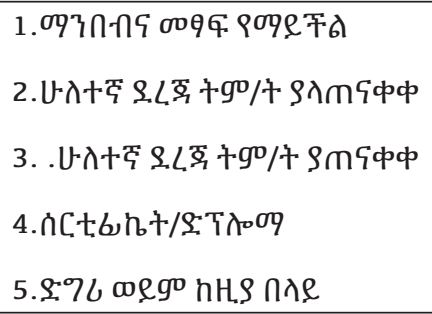 \\
\hline 06 & ९ก் Uらナ & 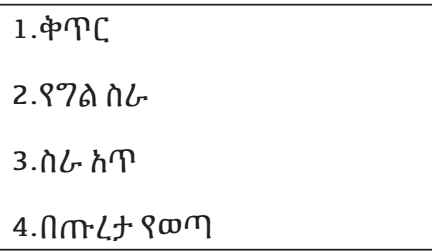 \\
\hline 07 & 97ח. Vלt & - \\
\hline 08 & pootes nt & $\begin{array}{l}1.7 \mathrm{mC} \\
2 . n+\infty 9\end{array}$ \\
\hline
\end{tabular}

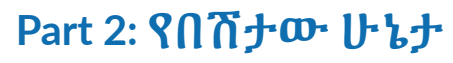

\begin{tabular}{|c|c|c|}
\hline 08 & 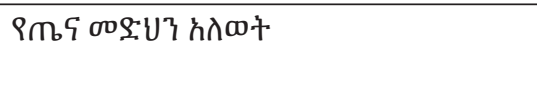 & $\begin{array}{l}1 . h \varphi \\
2 .\end{array}$ \\
\hline 09 & 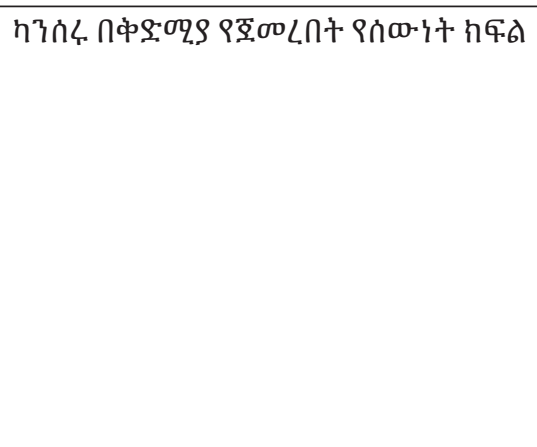 & 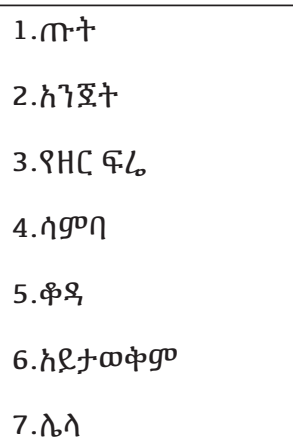 \\
\hline 010 & 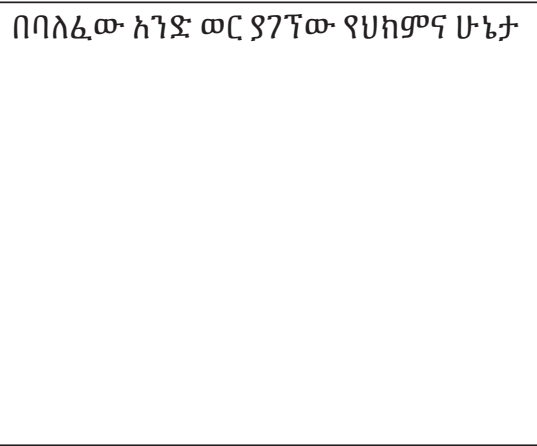 & 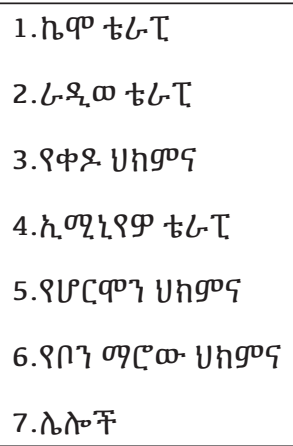 \\
\hline 011 & กగ゙๋ & ------------- \\
\hline
\end{tabular}




\begin{tabular}{|c|c|c|}
\hline 012 & 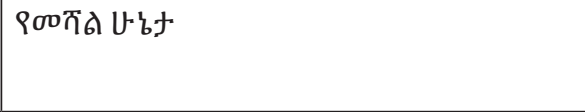 & $\begin{array}{l}1 . h \omega \\
2 . h \varrho \varrho \lambda g^{\circ}\end{array}$ \\
\hline 013 & 入 & $\begin{array}{l}1 . h \varphi p \\
2.9 \lambda g^{\circ}\end{array}$ \\
\hline 014 & $h \omega \cdot h \lambda \cdot 90 \eta 90 \eta$ & \\
\hline 015 & 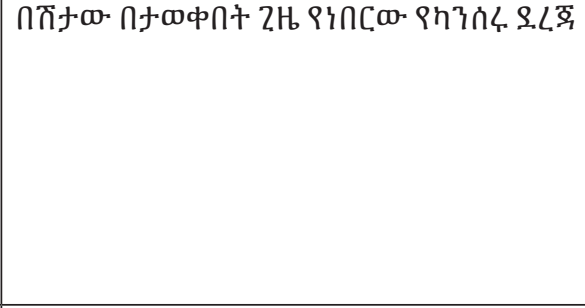 & 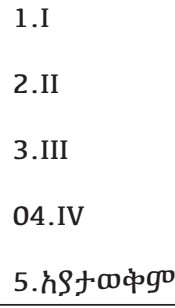 \\
\hline 016 & กד̃ & $\begin{array}{l}1 . h \lambda \omega \cdot \\
2.9 \lambda \omega \cdot g o\end{array}$ \\
\hline 017 & 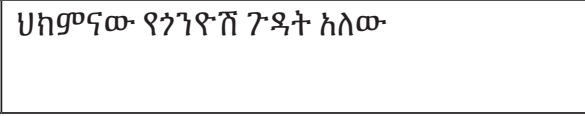 & $\begin{array}{l}1 . h \varphi \\
2.9 \lambda \omega . g 0\end{array}$ \\
\hline
\end{tabular}

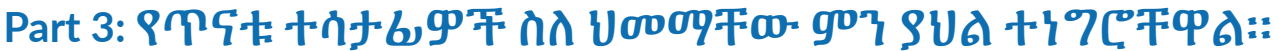

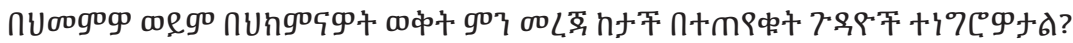

\begin{tabular}{|c|c|c|}
\hline +. $\$$ & T९qg市 & 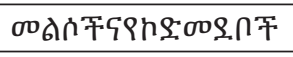 \\
\hline 01 & Uơgoggou hł & 1. h9 2. hด十々 \\
\hline 02 & 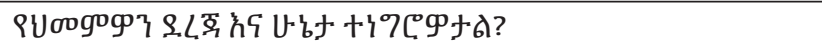 & 1. h9 2. hด十々 \\
\hline 03 & 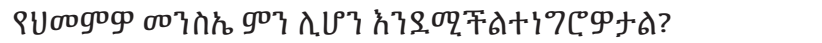 & 1. h9 2. hด十々 \\
\hline 04 & 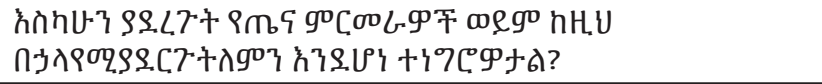 & 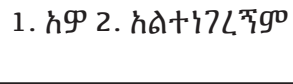 \\
\hline 05 & 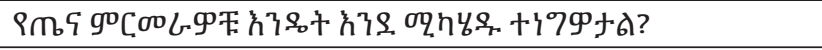 & 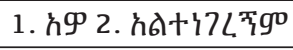 \\
\hline 06 & 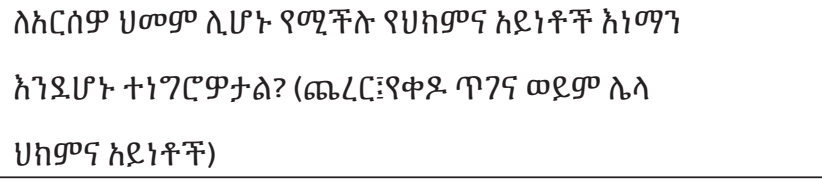 & 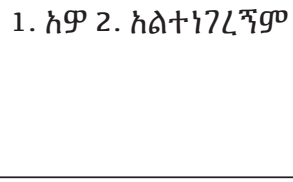 \\
\hline 07 & 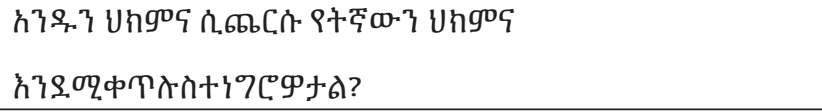 & 1. $h^{9} 2 . h \lambda+h 7 L^{\top} g^{g o}$ \\
\hline 08 & 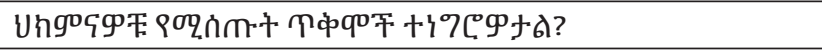 & 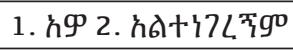 \\
\hline 09 & 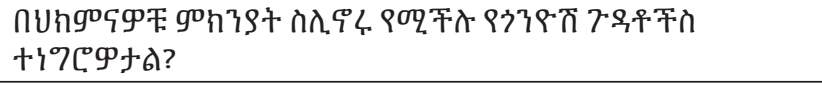 & 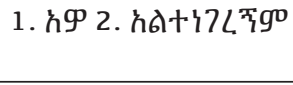 \\
\hline 010 & 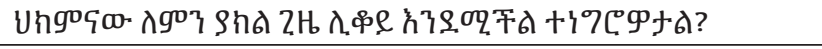 & 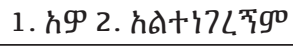 \\
\hline 011 & 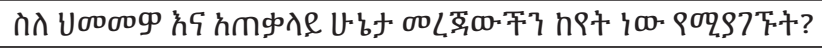 & 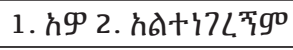 \\
\hline
\end{tabular}




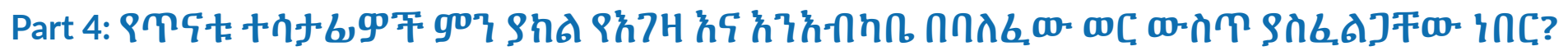

\begin{tabular}{|c|c|c|c|c|c|c|}
\hline \multirow[t]{5}{*}{ †.中. } & \multirow[t]{5}{*}{ 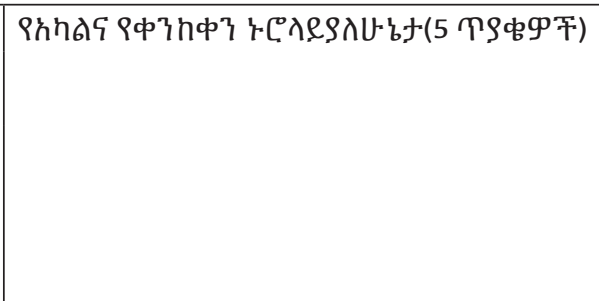 } & \multicolumn{5}{|c|}{ 9hs: oosn市 } \\
\hline & & \multicolumn{2}{|c|}{ 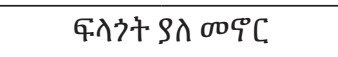 } & \multicolumn{3}{|c|}{ 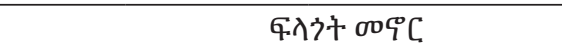 } \\
\hline & & อч宇9 & ६ìt & $+3 \pi$ & ovmis & n§+氶 \\
\hline & & $\varphi \lambda \xi g o$ & $+q^{0} \lambda+\lambda$ & 纺午 & ६i市 & 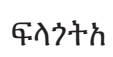 \\
\hline & & & & $h \lambda^{3}$ & $h \lambda^{\mathrm{F}}$ & $\lambda^{7}$ \\
\hline 1 & Uoogo & 1 & 2 & 3 & 4 & 5 \\
\hline 2 & 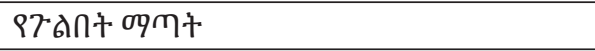 & 1 & 2 & 3 & 4 & 5 \\
\hline 3 & 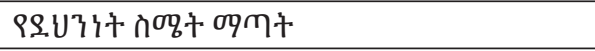 & 1 & 2 & 3 & 4 & 5 \\
\hline 4 & 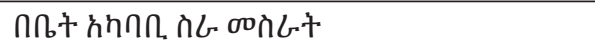 & 1 & 2 & 3 & 4 & 5 \\
\hline 5 & の0內 & 1 & 2 & 3 & 4 & 5 \\
\hline \multirow{4}{*}{\multicolumn{2}{|c|}{ 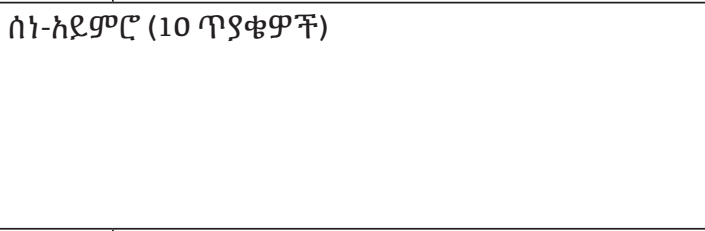 }} & \multicolumn{2}{|c|}{ 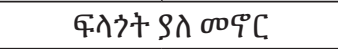 } & \multicolumn{3}{|c|}{ 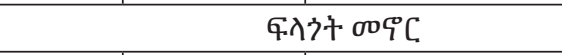 } \\
\hline & & ยบั79С & 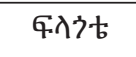 & +37 & ovmis & 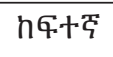 \\
\hline & & $9 \lambda_{\mathfrak{1}} g^{0}$ & 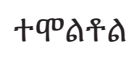 & 枋午 & ६入方 & ६へ斻 \\
\hline & & & & $h \lambda^{3}$ & $h \lambda \xi$ & $\lambda \overline{7}$ \\
\hline 1 & §CU† & 1 & 2 & 3 & 4 & 5 \\
\hline 2 & 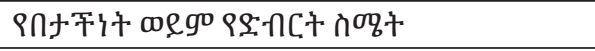 & 1 & 2 & 3 & 4 & 5 \\
\hline 3 & 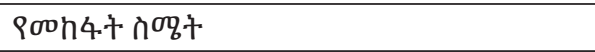 & 1 & 2 & 3 & 4 & 5 \\
\hline 4 & 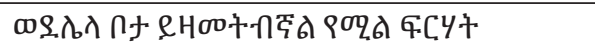 & 1 & 2 & 3 & 4 & 5 \\
\hline 5 & 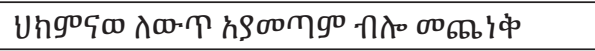 & 1 & 2 & 3 & 4 & 5 \\
\hline 6 & 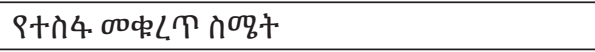 & 1 & 2 & 3 & 4 & 5 \\
\hline 7 & 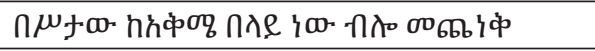 & 1 & 2 & 3 & 4 & 5 \\
\hline 8 & 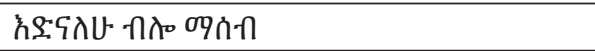 & 1 & 2 & 3 & 4 & 5 \\
\hline 9 & 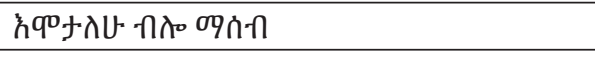 & 1 & 2 & & 4 & 5 \\
\hline 10 & ก̀n+ட.mL & 1 & 2 & 3 & 4 & 5 \\
\hline \multirow{4}{*}{\multicolumn{2}{|c|}{ 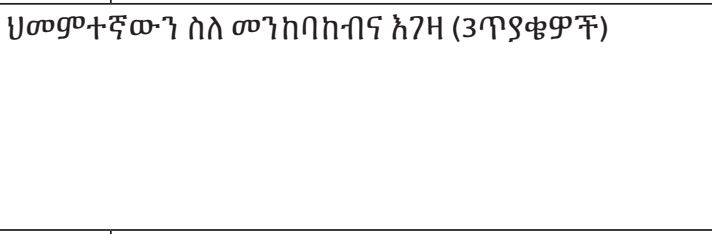 }} & \multicolumn{2}{|c|}{ ६तiे $9 \lambda 009 \mathrm{C}$} & \multicolumn{3}{|c|}{ Fìt 009} \\
\hline & & ८чтั7 & Fìt & +37 & 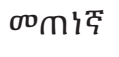 & n६+家 \\
\hline & & $\varphi \lambda^{\boldsymbol{z}} g^{\circ}$ & + $q^{0} \lambda+{ }^{\circ}$ & 舟公方 & 和方 & 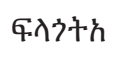 \\
\hline & & & & $h \lambda^{7}$ & $h \lambda^{\pi}$ & $\lambda^{3}$ \\
\hline 1 & 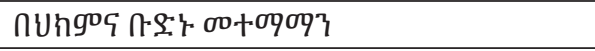 & 1 & 2 & 3 & 4 & 5 \\
\hline 2 & 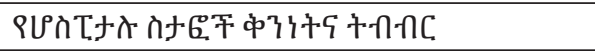 & 1 & 2 & 3 & 4 & 5 \\
\hline 3 & 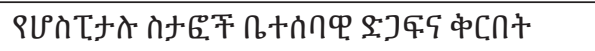 & 1 & 2 & 3 & 4 & 5 \\
\hline
\end{tabular}




\begin{tabular}{|c|c|c|c|c|c|c|}
\hline \multicolumn{2}{|c|}{ 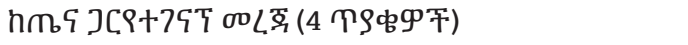 } & \multicolumn{2}{|c|}{ 舟方市 $9 \lambda 009 \mathrm{C}$} & \multicolumn{3}{|c|}{ §入う午 00 } \\
\hline & & ८ч⿱冖⿻ & & $+3 \pi$ & $\boldsymbol{\sigma o m}_{\mathrm{m}}$ & 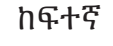 \\
\hline & & $\varphi \lambda_{\overline{3}} g^{0}$ & 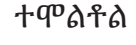 & ६ク方 & ६入斻 & ६入市h \\
\hline & & & & $h \lambda^{\top}$ & $h \lambda_{\bar{\gamma}}$ & $\lambda \overline{7}$ \\
\hline 1 & 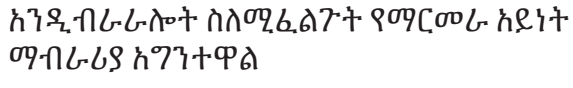 & 1 & 2 & 3 & 4 & 5 \\
\hline 2 & 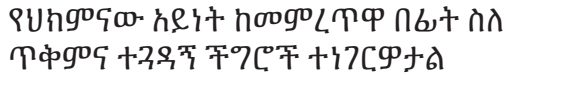 & 1 & 2 & 3 & 4 & 5 \\
\hline 3 & 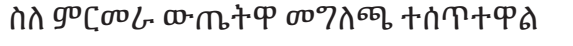 & 1 & 2 & 3 & 4 & 5 \\
\hline 4 & 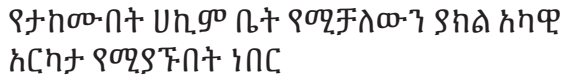 & 1 & 2 & 3 & 4 & 5 \\
\hline \multirow{4}{*}{\multicolumn{2}{|c|}{ 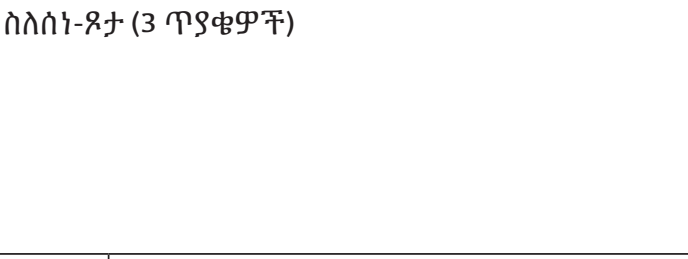 }} & \multicolumn{2}{|c|}{ 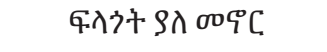 } & \multicolumn{3}{|c|}{ 六方年 00 } \\
\hline & & อчㅜำ & ६入ेt & $+3 \pi$ & 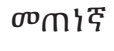 & n६+疋 \\
\hline & & $\varphi \lambda^{\pi} g^{\circ}$ & $+9^{0}$ 入๐ & ६入方 & ६入市 & ६入计么 \\
\hline & & & & $h \lambda^{\Im}$ & $h \lambda \bar{z}$ & $\lambda \boldsymbol{z}$ \\
\hline 1 & 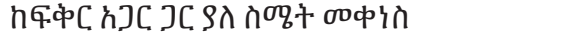 & 1 & 2 & 3 & 4 & 5 \\
\hline 2 & 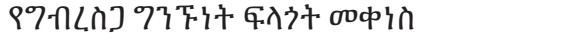 & 1 & 2 & 3 & 4 & 5 \\
\hline 3 & ก่ & 1 & 2 & 3 & 4 & 5 \\
\hline
\end{tabular}

९+\%o G.

b.

中?

९m९̨фa. ǹgo

b.

中? 